Modelagem de dados de sistemas reparáveis com fragilidade 



\title{
Modelagem de dados de sistemas reparáveis com fragilidade
}

\author{
Cirdêmia Costa Feitosa
}

Orientadora: Profa. Dra. Vera Lucia D. Tomazella

Dissertação apresentada ao Instituto de Ciências Matemáticas e de Computação - ICMC-USP e ao Departamento de Estatística - DEs-UFSCar, como parte dos requisitos para obtenção do título de Mestre em Estatística - Interinstitucional de Pós-Graduação em Estatística. VERSÃO REVISADA

\section{USP/UFSCar - São Carlos}

Outubro de 2015 
Ficha catalográfica elaborada pela Biblioteca Prof. Achille Bassi e Seção Técnica de Informática, ICMC/USP, com os dados fornecidos pelo(a) autor(a)

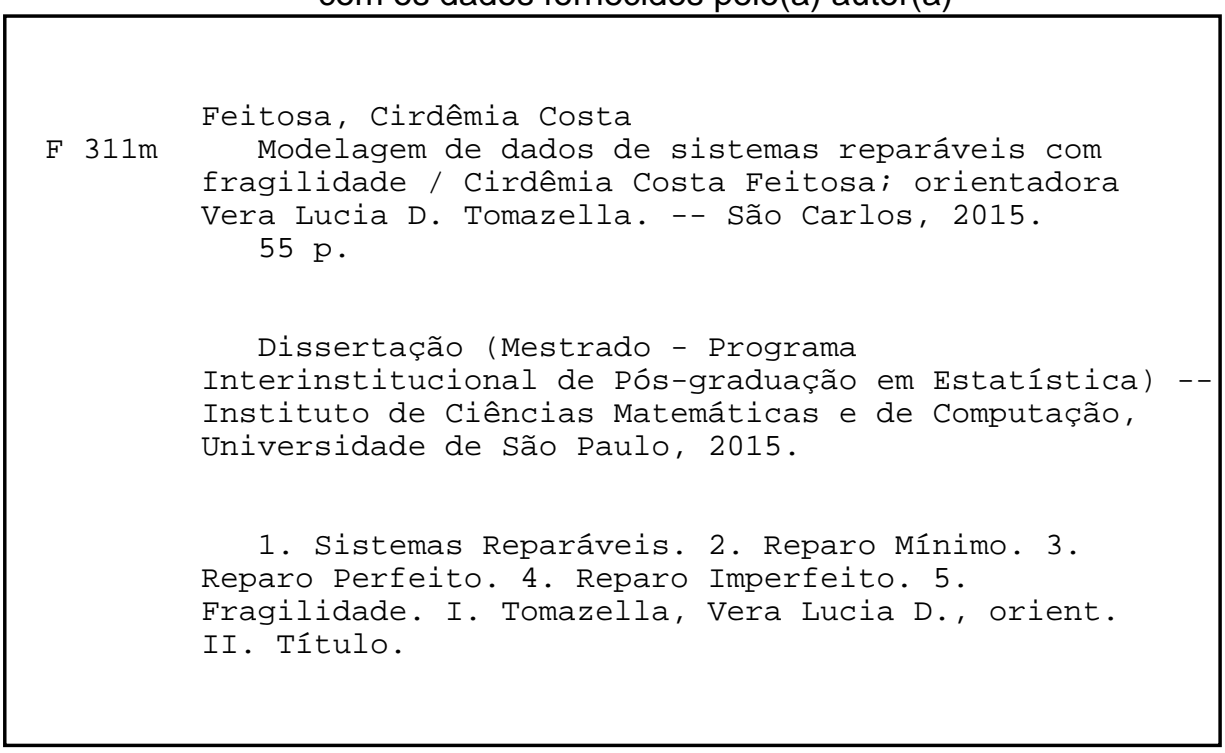




\title{
Modeling repairable systems data with fragility
}

\author{
Cirdêmia Costa Feitosa
}

Advisor: Profa. Dra. Vera Lucia D. Tomazella

\begin{abstract}
Master dissertation submitted to the Instituto de Ciências Matemáticas e de Computação - ICMC-USP and to the Departamento de Estatística - DEs-UFSCar, in partial fulfillment of the requirements for the degree of the Master Joint Graduate Program in Statistics DEs-UFSCar/ICMC-USP. FINAL VERSION.
\end{abstract}

\section{USP/UFSCar - São Carlos October 2015}





\section{Dedicatória}

Dedico este trabalho ao meu querido companheiro Leone Maia de Souza, presente nas minhas conquistas e nas minhas dificuldades. Dedico também a minha mãe Marlene Costa Feitosa, sem o seu apoio esta conquista não seria possível. 



\section{Agradecimentos}

A Profa. Dra. Vera Lucia D. Tomazella pela paciência na orientação do trabalho, ao Prof. Dr. Afrânio M. C. Vieira pelo auxílio na construção do conhecimento, a minha amiga Amanda Morales Eudes pela ajuda e pelo apoio constante. 



\section{Resumo}

Os modelos de sistemas reparáveis usuais são os de reparo mínimo, perfeito e imperfeito, sendo que, na literatura, o modelo de reparo mínimo é o mais explorado. Em sistemas reparáveis é comum que componentes do mesmo tipo sejam estudados e nestes casos é relevante verificar a heterogeneidade entre eles. Segundo Vaupel et al. (1979), os métodos padrões em análise de dados de sistemas reparáveis ignoram a heterogeneidade não observada e em alguns casos esta deveria ser considerada. Tal variabilidade pode ser estimada a partir dos modelos de fragilidade, caracterizados pela utilização de um efeito aleatório. Propõe-se o modelo de reparo mínimo com fragilidade, a fim de estimar a heterogeneidade não observada entre sistemas. Para este modelo foi realizado um estudo de simulação com o objetivo de analisar as propriedades frequentistas do processo de estimação. A aplicação em um conjunto de dados reais mostrou a aplicabilidade do modelo proposto, em que a estimação dos parâmetros foram determinadas à partir das abordagens de máxima verossimilhança e Bayesiana.

Palavras-chave: Sistemas Reparáveis, Reparo Mínimo, Reparo Perfeito, Reparo Imperfeito, Fragilidade, Bayesiana. 



\begin{abstract}
The usual models in repairable systems are minimal, perfect and imperfect repair, and,in the literature, the minimum repair model is the most explored. In repairable systems it is common that the same type of components are studied and in these cases is relevant to verify the heterogeneity between them. According to Vaupel et al. (1979), the standard methods for analysis of repairable systems data ignore the heterogeneity not observed and in some cases this should be considered. Such variability can be estimated from frailty models, characterized by using a random effect. It is proposed that the minimum repair model with frailty in order to estimate the heterogeneity not observed between systems. For this model it was conducted a simulation study in order to analyze the frequentist properties of the estimation process. The application of a real data set showed the applicability of the proposed model, in which the estimation of the parameters were determined from maximum likelihood and Bayesian approaches.
\end{abstract}

Keywords: Repairable Systems, Minimal Repair, Perfect Repair, Imperfect Repair, Frailty, Bayesian. 



\section{Sumário}

Lista de Tabelas

Lista de Figuras $\quad$ v

1 Introdução 1

1.1 Motivação . . . . . . . . . . . . . . . . . . . . . 3

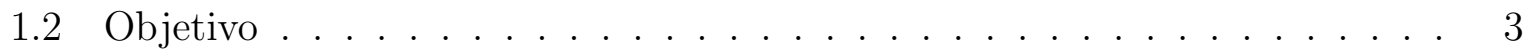

1.3 Organização dos Capítulos . . . . . . . . . . . . . . . . . 4

2 Sistemas Reparáveis 5

2.1 Conceitos Básicos de Processo de Contagem . . . . . . . . . . . . . 6

2.2 Modelos de Sistemas Reparáveis . . . . . . . . . . . . . . . . . . . . . . . . 9

2.2.1 Reparo Mínimo . . . . . . . . . . . . . . . . . . . . . 9

2.2.2 Reparo Perfeito . . . . . . . . . . . . . . . . 10

2.2 .3 Reparo Imperfeito . . . . . . . . . . . . . . . . . . . . . . 11

2.3 Inferência . . . . . . . . . . . . . . . . . . . . . . . 14

2.4 Estudo de Simulação para o modelo ARA . . . . . . . . . . . . . . . . 23

2.5 Aplicação em dados reais . . . . . . . . . . . . . . . . . . . . 25

2.6 Alguns comentários . . . . . . . . . . . . . . . . . . . . . . . . . . . . 29

3 Modelo de Reparo Mínimo com fragilidade $\quad 31$

3.1 Modelo de fragilidade multiplicativo . . . . . . . . . . . . . . . . 32

3.2 Estudo de Simulação . . . . . . . . . . . . . . . . . . . . . . . . 38

3.3 Aplicação aos dados dos caminhões basculantes . . . . . . . . . . . . . . . 40

3.4 Alguns comentários . . . . . . . . . . . . . . . . . . . . . . 42 
4 Método de estimação bayesiano

4.1 Aplicação em dados reais . . . . . . . . . . . . . . . . . . . . 45

4.1.1 Estimação à posteriori da a v.a. Z . . . . . . . . . . . . . . . 48

4.2 Alguns comentários . . . . . . . . . . . . . . . . . 50

5 Considerações finais e propostas futuras $\quad 51$

Referências Bibliográficas $\quad 53$ 


\section{Lista de Tabelas}

2.1 Resultados do estudo de simulação para o Modelo ARA . . . . . . . . . . . . 25

2.2 Esboço dos tempos de falha dos caminhões basculantes . . . . . . . . . . . . 25

2.3 Estimativas obtidas pelo Método Clássico de Máxima Verossimilhança . . . . 27

3.1 Resultados do estudo de simulação do modelo de RM com fragilidade . . . . . . 39

3.2 Estimativas para os parâmetros do modelo de RM com fragilidade via Máxima Verossimilhança e bayesiano . . . . . . . . . . . . . . . . . . 40

3.3 Critérios AIC, BIC e Log-verossimilhança dos modelos . . . . . . . . . . . 41

4.1 Estimativas obtidas pelo Método de Estimação Bayesiano . . . . . . . . . . . . 47

4.2 Mediana à posteriori simulados para a variável $Z$. . . . . . . . . . . . . 48

4.3 Média à posteriori para a variável $Z \ldots \ldots$. . . . . . . . . . . . . 50 



\section{Lista de Figuras}

2.1 Funções de intensidade pelo PLP para modelos ARA e ARI. . . . . . . . . 14

2.2 Gráfico dos Resultados da Simulação para o Modelo ARA . . . . . . . . . 26

2.3 Gráfico de comparação de KM com função de confiabilidade estimada . . 29

3.1 Gráfico dos Resultados da Simulação para o Modelo de RM com fragilidade 39

3.2 Gráfico de comparação de KM com função de confiabilidade estimada para o modelo de RM com fragilidade . . . . . . . . . . . . . . . . . . . 41

4.1 Convergência dos parâmetros $\beta$ e $\eta$ do modelo de RM . . . . . . . . . . . 46

4.2 Convergência dos parâmetros $\beta, \eta$ e $\alpha$ do modelo de RM com fragilidade . 47

4.3 Função de intensidade condicionada à fragilidade para cada caminhão . . . 49 



\section{Capítulo 1}

\section{Introdução}

Um dos principais problemas encontrados nas atividades realizadas em série é a ocorrência de falhas, por exemplo, falhas ocorridas em esteiras em empresas de engenharia de fabricação, em veículos de transporte de empresas de logística, em componentes de softwares, avaria em equipamentos complexos como painéis de controle ou ainda problemas em caminhões de frotas de transportadoras.

Tais falhas podem gerar para as empresas custos excedentes ao orçamento, atrasos na entrega de seus produtos ou da prestação de serviços e até perda de mercadorias e de clientes. Assim, ocorrida a indesejada falha, é imprescindível que o processo inicialmente planejado seja retomado e para isso, uma ação de reparo deve ocorrer o quanto antes e da melhor maneira possível.

O tipo de reparo realizado pode colaborar para a recorrência das falhas, pois, se o reparo for simples, pode-se pensar que o problema irá reaparecer em um tempo relativamente curto e se o reparo for mais completo, é plausível pensar que o equipamento levará mais tempo para falhar novamente. Entretanto, um reparo simples pode ser mais rápido e, consequentemente, mais apropriado dependendo da urgência da retomada das atividades, enquanto que um reparo mais completo pode levar mais tempo que o desejado. Desta forma, estudar o efeito dos reparos é de grande interesse para o aprimoramento do planejamento das empresas.

Esta análise é possível à partir do estudo dos modelos de Sistemas Reparáveis, em que pode-se mensurar, por exemplo, o risco de um equipamento falhar considerando o seu histórico de falhas e também pode-se estimar o efeito de um reparo. Ascher e Feingold (1984) definem que um sistema é considerado reparável se este falhar e voltar a operar satisfatoriamente após uma ação de reparo, sem que haja a necessidade de substituir o 
sistema como um todo.

Rigdon e Basu (2000) exemplificam a diferença entre os sistemas reparáveis e sistemas não reparáveis, sendo que os reparáveis podem e devem ser restaurados, como problemas em automóveis, e os não reparáveis não podem ser restaurados ou não é conveniente o reparo, como uma lâmpada ou uma calculadora de bolso.

Na literatura, os modelos usuais de sistemas reparáveis são os de reparo mínimo, perfeito e imperfeito e Toledo (2014) diz que estes modelos devem ser capazes de descrever a ocorrência de falhas ao longo do tempo, bem como o efeito das manutenções corretivas.

O reparo mínimo é a suposição mais explorada na literatura, que concentra-se em corrigir apenas o componente originador da falha, deixando o sistema na mesma condição em que estava anterior à ela.

Já a suposição de reparo perfeito pode ser razoável para sistemas com uma unidade que seja estruturalmente simples (Kijimạ, 1989). Sob tal suposição, o reparo é feito de maneira que o torne tão bom quanto novo. Neste caso, o processo que descreve a ocorrência de falhas é o Processo de Renovação, em que a probabilidade de falha se inicia a cada ação de reparo.

Entretanto, na prática as atividades de reparo nem sempre ocorrem em situações extremas, mas sim de maneira intermediária, ou seja, após a ação de reparo o sistema estará numa condição melhor que a anterior à falha, mas não pode ser considerado como novo.

Um modelo sugerido por Brown e Proschan (1983) assume que no tempo de cada falha, um reparo perfeito ocorre com probabilidade $p$ e um reparo mínimo ocorre com probabilidade (1-p), independentemente do histórico de falhas. Esse modelo é um simples exemplo do que pode se chamar de modelo de Reparo Imperfeito (RI), para $0<p<1$ que posteriormente foi generalizado em diversas direções (Lindqvist 2006).

Kijima (1989) introduziu a ideia de idade virtual do sistema, que é representada por uma função positiva da idade real e do histórico de falhas. Este modelo tem um parâmetro denotado por $\theta$, o qual representa o grau de eficiência do reparo e, assim, quando $0<\theta<1$ o reparo é o imperfeito e os casos especiais são para $\theta=1$, cujo reparo é mínimo e para $\theta=0$, em que o reparo é perfeito.

Em análise de dados de sistemas reparáveis é comum que muitos componentes do mesmo tipo sejam estudados e nestes casos é relevante verificar a heterogeneidade entre os sistemas. Proschan (1963) apontou que a heterogeneidade não observada pode explicar 
taxas de falhas crescentes, o que é frequentemente encontrado em análise de confiabilidade.

A heterogeneidade não observada pode ser estimada a partir dos modelos de fragilidade. Este modelo é caracterizado pela utilização de um efeito aleatório, isto é, uma variável aleatória não observável que representa as informações que não puderam ou não foram observadas.

Segundo Vaupel et al. (1979), os métodos padrões em análise de dados de sistemas reparáveis ignoram a heterogeneidade não observada. Sendo assim, considera-se que os sistemas tenham diferentes fragilidades e aqueles que forem mais frágeis, falharão mais cedo que os demais. Esta é a principal abordagem deste trabalho.

\subsection{Motivação}

O trabalho foi motivado por um estudo feito por Toledo (2014) relacionado à problemas de manutenção em caminhões de uma mineradora brasileira. Um destes estudos refere-se à caminhões basculantes não projetados para operarem nas condições encontradas em locais de mineração, mas auxiliam no processo como um todo. Quando estes caminhões apresentam falhas, muitas vezes o seu reparo é imediato, pois sua finalidade é dar o auxílio aos caminhões de grande porte projetados para o transporte de minério.

No estudo realizado por Toledo (2014), os tempos de falha entre os distintos caminhões foram considerados independentes. Acredita-se que é relevante verificar se há heterogeneidade entre os sistemas, pois podem existir informações tais como condições ambientais, estresse de uso, entre outras, que não puderam ou não foram incluídas no estudo, mas que de alguma forma são importantes para a análise deles.

O interesse é caracterizar a fragilidade destes caminhões, isto é, quanto mais frágil for o caminhão, mais vezes ele irá apresentar o evento de interesse, que neste caso significa que quanto mais reparos o caminhão sofrer, mais vezes ele irá falhar em relação aos demais.

\subsection{Objetivo}

A proposta deste trabalho é abordar os modelos usuais de sistemas reparáveis, sendo eles o modelo de reparo mínimo, reparo perfeito e reparo imperfeito que inclui as classes de modelos de sistemas reparáveis ARI (Aritimetic Reduction of Intensity) e ARA (Arithmetic Reduction of Age) considerando apenas os modelos de memória 1. Através de um 
estudo de simulação para o modelo ARA pretende-se verificar as propriedades frequentistas para tal modelo, bem como realizar uma aplicação em um conjunto de dados reais a partir da abordagem clássica pelo método de máxima verossimilhança.

A fim de quantificar a heterogeneidade não observável entre os sistemas, propõe-se o modelo de reparo mínimo com fragilidade, em que um termo de fragilidade é inserido à função de intensidade do modelo de reparo mínimo sob o processo de lei de potência em que supõe-se que este termo segue uma distribuição gama. São consideradas as abordagens clássica e bayesiana para a estimação dos parâmetros do modelo proposto.

\subsection{Organização dos Capítulos}

No Capítulo 2, são apresentados os modelos usuais de sistemas reparáveis, iniciando pelo modelo de reparo mínimo, em que são descritos os conceitos básicos de processo de contagem e a forma paramétrica do processo de lei de potência. Na sequência, apresentase o modelo de reparo perfeito e as classes de modelo ARA e ARI do reparo imperfeito, além disso, são apresentados os resultados de um estudo de simulação realizado para o modelo ARA e os resultados de uma aplicação feita em um conjunto de dados reais, cujas estimativas foram obtidas a partir do método de máxima verossimilhança para o reparo mínimo, ARA e ARI.

Tendo em vista a possibilidade de haver heterogeneidade não observável entre os sistemas, propõe-se no Capítulo 3 o modelo de reparo mínimo com fragilidade multiplicativa. Neste capítulo, os principais conceitos do modelo de fragilidade multiplicativo são apresentados, assim como um estudo de simulação para verificar as propriedades frequentistas do modelo em questão e para ilustrar a aplicabilidade do modelo proposto, um conjunto de dados reais foi utilizado.

No Capítulo 4 apresenta-se uma abordagem bayesiana para a estimação dos parâmetros do modelo de reparo mínimo e do modelo de reparo mínimo com fragilidade. 


\section{Capítulo 2}

\section{Sistemas Reparáveis}

Uma definição comumente utilizada na literatura para definir sistemas reparáveis foi dada por Ascher e Feingold (1984), de que um sistema, sejam eles máquinas, equipamentos eletrônicos ou softwares, é considerado reparável se após uma falha, sua atividade puder ser retomada satisfatoriamente por meio de reparo, sem a necessidade de substituição do sistema como um todo. Os autores relataram que os pesquisadores e profissionais de confiabilidade não reconheciam a diferença crucial entre os sistemas reparáveis e não reparáveis e demostraram com vários exemplos simples, como conclusões erradas podem ser tomadas se essa diferença não for considerada. Conforme observado por Lindqvist (2006), o livro Ascher e Feingold (1984) parece ser o primeiro dedicado exclusivamente à confiabilidade dos sistemas reparáveis e durante muito tempo esta foi a principal referência na literatura e ainda é uma das principais fontes de citação.

Rigdon e Basu (2000) é outra referência importante nesta área, pois aborda essa crucial diferença entre os sistemas reparáveis e não reparáveis. Um sistema reparável é aquele que pode ser restaurado após uma falha, desde que seu reparo seja compensável, como concertos em automóveis ou em equipamentos eletrônicos mais complexos, por exemplo, geladeiras e televisores. Os sistemas não reparáveis são aqueles que são descartados após uma falha, como ocorre com lâmpada queimadas, ou o sistema pode até ser passível de reparo, mas seu custo é similar à um novo equipamento e então compensa o descarte, como é o caso de calculadoras e alguns problemas em telefones celulares.

É importante ressaltar a diferença entre censura e truncamento, pois estes conceitos são diferentes e ambos não devem ser confundidos, apesar de terem alguma semelhança.

Em sistemas não reparáveis o conceito de censura é devido para a falta de informação a respeito da variável de interesse. A censura ocorre quando se tem alguma informação sobre 
a variável aleatória de interesse, mas esta informação é incompleta. Existem basicamente três tipos de censura: à direita, à esquerda e intervalar; e três mecanismos de censura: censura tipo I, censura tipo II e censura aleatória.

Na censura tipo I, o estudo é conduzido até um tempo pré-fixado enquanto que na censura tipo II o estudo é conduzido até que um certo número de eventos de interesse ocorram. Já a censura intervalar ocorre quando a unidade observada abandona o estudo sem que tenha apresentado o evento de interesse

Em sistemas reparáveis o conceito utilizado é o de truncamento e há, basicamente, duas maneiras de se observar os dados. Na primeira, a coleta de dados termina em um limite de tempo pré estabelecido $t$, denominado por truncamento por tempo, e na segunda, a coleta de dados termina após a ocorrência de um número predeterminado de falhas, denotado por truncamento por falha.

Portanto, no caso em que as amostras apresentaram o fenômeno de censura, todos os casos são registrados, mesmo aqueles que são tidos como censurados. Por outro lado, nas amostras truncadas, os dados registrados são apenas aqueles que ocorrem dentro do limite de truncamento e os demais são omitidos ou descartados.

Dessa forma, tanto a censura quanto o truncamento resultam em falta de informação sobre a variável de interesse, no entanto, é necessário destacar que a principal diferença é que no caso de censura há o registro dos dados e no caso do truncamento não há.

Nos sistemas não reparáveis, a unidade é observada até a ocorrência do evento de interesse, por outro lado, em sistemas reparáveis, após a ocorrência da falha o sistema é reparado e continua sendo observado. Na modelagem, a suposição implícita é que o sistema é reparado e imediatamente volta a operar (Lindqvist, 2006), ou seja, o tempo de reparo é considerado tão pequeno que pode ser desprezado.

\subsection{Conceitos Básicos de Processo de Contagem}

Suponha que $T$ é a variável aleatória que representa o tempo de falha, sendo que o experimento começa em $t=0$. Os eventos ocorrem aleatoriamente, sendo que $T_{1}$ ocorre primeiro, $T_{2}$ ocorre segundo e assim sucessivamente, então, $T_{i}$ denota o tempo em que ocorre o $i$-ésimo evento. Se $\mathrm{N}(\mathrm{t})$ representar o número de eventos total ocorridos no intervalo $(0, t)$ este é dito processo de contagem.

Um processo de contagem é um processo estocástico que descreve a ocorrência de 
determinados eventos no tempo e deve satisfazer as seguintes propriedades:

- $N(t) \geq 0$ e $N(0)=0$;

- $N(t)$ é um número inteiro;

- $t \longmapsto N(t)$ é contínua à direita;

- $\Delta N(t)=\lim _{h \rightarrow 0} N(t)-N(t-h)=0$.

A distribuição Poisson é uma distribuição discreta muito utilizada e desempenha um papel importante no estudo de processo de Poisson. Uma variável aleatória $X$ tem distribuição Poisson se sua função distribuição de probabilidade for

$$
p(x)=P(X=x)=\frac{\phi^{x} \mathrm{e}^{-\phi}}{x !}
$$

para $x=0,1,2, \ldots$ e $\phi>0$, podendo ser denotada por $X \sim P O I(\phi), \operatorname{com} E(X)=\phi$ e $V(X)=\phi$.

O processo de Poisson que pode ser homogêneo ou não homogêneo. Um processo $N(t)$ é dito ser um processo de Poisson homogêneo (PPH) com intensidade $\lambda>0$ se:

- $N(0)=0$;

- $N(t)$ tem incrementos estacionários independentes;

- $\lim _{h \rightarrow 0} \frac{P(N(t+h)-N(t)=1)}{h}=\lambda$.

Assim, no PPH a $E[N(t)]=\lambda$ e $\operatorname{Var}[N(t)]=\lambda$. Então, para intervalos unitários, o número médio de eventos nestes intervalos é $\lambda$. Além disso, os tamanhos dos intervalos de tempos $\left\{Z_{n} ; n \geq 1\right\}$ de um PPH com intensidade $\lambda$ são variáveis aleatórias com distribuição exponencial com taxa $\lambda$ independentes entre si. Por fim, o número de eventos que ocorrem em um intervalo de tempo de comprimento $t$ é uma variável aleatória discreta de Poisson com taxa $\lambda$.

O PPH ocorre quando os incrementos são estacionários, assim, a distribuição do número de eventos em um intervalo depende apenas do comprimento do intervalo, em outras palavras, a taxa de intensidade do processo será sempre $\lambda$ para todos os tempos.

Então, o PPH deve ser aplicado com cautela, pois, uma vez que a função de intensidade é constante, esta não pode ser utilizada para modelar sistemas que estão deteriorando ou em processo de melhoria. 
O PPNH é um processo de Poisson cuja a função de intensidade não é constante. Um processo $N(t)$ é dito ser um PPNH com função intensidade $\lambda(t)>0$ se:

1. $N(0)=0$;

2. $N(t)$ tem incrementos independentes, isto é, se $a<b \leq c<d$, então as variáveis aleatórias $N(b)-N(a)$ e $N(d)-N(c)$ são independentes;

3. Há uma função $\lambda$ tal que

$$
\lim _{h \rightarrow 0} \frac{P(N(t+h)-N(t)=1)}{h}=\lambda(t)
$$

A partir dessas propriedades tem-se que:

- $N(t) \sim$ Poisson $\left(\Lambda(t)=\int_{0}^{t} \lambda(u) d u\right)$

- $N(b)-N(a) \sim$ Poisson $(\Lambda(b)-\Lambda(a))$

Considerando estas definições e propriedades, pode-se agora definir a função de intensidade de falha. Então, assumindo que as falhas nos sistemas reparáveis são equivalentemente definidas pelo processo $\{N(t)\}_{t \geq 0}$ ou $\left\{T_{N(t)}\right\}_{t \geq 0}$, nos quais $N(t)$ denota o número de falhas até o tempo $t$ e $T_{N(t)}$ corresponde ao tempo decorrido até a $N(t)$-ésima falha, pode-se, então, definir a função intensidade de falha que é dada por:

$$
\rho(t)=\lim _{\Delta t \rightarrow 0} \frac{P\left(N(t+\delta t)-N(t)=1 \mid v_{t}-\right)}{\Delta t},
$$

para todo $t>0$, onde $v_{t}$ - é o histórico de todos os tempos de falha ocorridos antes de $t$.

Determina-se a função média, que é a função de intensidade acumulada da função $(2.3)$, por

$$
\Lambda(t)=E[N(t)]=\int_{0}^{t} \lambda(u) d u
$$

Uma forma paramétrica muito utilizada em sistemas reparáveis é o Processo de Lei de Potência. Seja $N(t)$ o número de falhas desde o início do acompanhamento do sistema até um certo tempo $t$, então, se $N(t)$ segue um Processo de Lei de Potência (PLP), a função de intensidade e a função intensidade acumulada, proposta por Crow (1974), são 
dadas, respectivamente, por

$$
\lambda(t)=\frac{\beta}{\eta}\left(\frac{t}{\eta}\right)^{\beta-1}
$$

$\mathrm{e}$

$$
\Lambda(t)=\left(\frac{t}{\eta}\right)^{\beta}
$$

em que $\eta>0$ é o parâmetro de escala e $\beta>0$ é o parâmetro de forma.

Segundo Oliveira et al. (2014), o parâmetro $\eta$ pode ser interpretado como o tempo durante o qual se espera exatamente que uma falha ocorra, ou seja, $E[N(\eta)]=1$, enquanto que $\beta$ representa a elasticidade do número esperado de falhas em relação ao tempo, então, quando $\beta>1$, o sistema está deteriorando.

De acordo com Crow (1974), este processo é usual na literatura de sistemas reparáveis e Oliveira et al. (2014) menciona que sua popularização é devida ao fato de sua função ser flexível. Mais precisamente, o PLP pode acomodar tanto intensidades de ocorrências crescentes, que ocorrem quando $\beta>1$, quanto intensidades decrescentes, $\beta<1$, ou ainda quando a intensidade de ocorrência do evento de interesse é constante, para os casos em que $\beta=1$, caracterizando um Processo de Poisson Homogêneo (PPH).

\subsection{Modelos de Sistemas Reparáveis}

O tipo de reparo a ser feito depende do tipo de sistema e, evidentemente, do tipo de avaria apresentada ou ainda do quão urgente o equipamento deve retomar suas atividades. Assim, nesta seção serão abordados os três modelos já mencionados.

\subsubsection{Reparo Mínimo}

Conforme mencionado anteriormente, o Reparo Mínimo (RM) é a suposição mais explorada na literatura. O RM concentra-se em corrigir apenas o componente originador da falha, deixando o sistema na mesma condição em que estava anterior à ela, conhecida na literatura por As Bad as Old - ABAO.

Tome como exemplo um secador de cabelo que está com mal funcionamento na ventoinha, problema normalmente causado pelo excesso de fios de cabelo que vão se acumulando 
ao longo do tempo. Então, supondo que seja este o único problema, basta remover este excesso e o secador voltará a funcionar normalmente. Este é um caso de reparo mínimo, que manteve o sistema nas condições anteriores à falha. Segundo Kijima (1989), esta suposição é plausível para sistemas constituídos de diversos componentes, tendo cada um o seu próprio modo de falha.

Quando supõe-se reparo mínimo, o processo associado à ocorrência de falhas pode ser descrito por um Processo de Poisson Não-Homogêneo (PPNH), conforme descrito na Seção (2.1), que é um caso particular de um processo de contagem, em que a probabilidade de falha em um pequeno intervalo de tempo não depende do seu histórico de falhas, mas apenas da idade do sistema, (Muralidharan 2008) e assim, a função de intensidade irá depender apenas do tempo.

Dessa maneira, define-se o modelo de RM, cuja função de intensidade é dada por

$$
\rho_{R M}(t)=\lambda(t)
$$

Sob o PLP, a função (2.7) fica igualmente à função (2.5), isto é

$$
\rho_{R M}(t)=\frac{\beta}{\eta}\left(\frac{t}{\eta}\right)^{\beta-1} .
$$

Isso significa que a intensidade do processo imediatamente depois da falha é a mesma encontrada imediatamente antes da falha e então, é exatamente como ele poderia ser se a falha não tivesse ocorrido, (Lindqvist, 2006). Na prática um reparo mínimo usualmente corresponde reparar ou repor somente a menor parte do sistema.

\subsubsection{Reparo Perfeito}

Tomando novamente o secador de cabelo como exemplo, considere que agora o problema está na resistência elétrica e para repará-lo, optou-se pela troca da resistência. Como este componente é um dos mais importantes para o seu funcionamento, o secador pode ser considerado como novo, ou seja, o reparo deixou este eletrônico numa condição melhor do que estava anterior à falha, o que caracteriza um tipo de reparo perfeito, pois deixou o secador numa condição conhecida na literatura como (As good as new - AGAN) tão boa quanto nova.

Segundo Kijima (1989), assumir reparo perfeito pode ser razoável para sistemas com 
uma unidade em que sua estrutura seja simples e, neste caso, o processo de falha é modelado por um processo de renovação.

O processo de renovação pode ser definido pelas distribuições conjuntas do processo de contagem $N(t)$. Este processo é dito renovável porque a probabilidade de falha se inicia a cada processo de reparo. A caracterização mais simples é através dos intervalos entre os tempos de ocorrências de falhas, sendo independente e identicamente distribuídos (i.i.d). Assim, se todas as variáveis aleatórias $Z_{n}$ são (i.i.d), então, $\left\{Z_{n} ; n \geq 1\right\}$ é um processo de renovação, em que $Z_{i}=T_{i}-T_{i-1}$ e $T_{0}=0$ que denota o tempo entre os eventos $n-1$ e $n$.

A principal razão para o estudo de processos de renovação é que em muitos processos complicados há determinados instantes em que o sistema retorna a um estado probabilisticamente equivalente ao estado inicial de maneira aleatória. Estes tempos de renovação incorporados permitem separar o comportamento a longo prazo do processo de ocorrência de falha, a partir do comportamento do processo real dentro de um período de renovação, que pode ser estudado através da teoria de renovação.

A função de intensidade de falhas do modelo de Reparo Perfeito (RP) é definida por

$$
\rho(t)=\lambda\left(t-T_{N(t)}\right)
$$

Sob o PLP, cuja função de intensidade é a (2.5), a função de intensidade de falhas para o RP é dada por

$$
\rho_{R P}(t)=\frac{\beta}{\eta}\left(\frac{t-T_{N(t)}}{\eta}\right)^{\beta-1}
$$

onde $T_{N(t)}$ é o tempo decorrido desde o início do sistema até a $N(t)$-ésima falha.

\subsubsection{Reparo Imperfeito}

Seguindo o exemplo do secador de cabelo com problemas na resistência elétrica, optouse por, ao invés de trocar a resistência, realizar um tipo de emenda (solda) no local onde houve a ruptura do fio dessa resistência. Esta ação de reparo representa um tipo de reparo imperfeito, cuja condição não é similar a de um novo secador, porém, a condição é melhor que a anterior à falha.

Vê-se que na prática, as ações de reparo nem sempre ocorrem em situações similares ao reparo mínimo ou reparo perfeito, pois em algumas situações o reparo deixa o equipamento 
em condições intermediárias.

Segundo Kijima et al. (1988) para sistemas compostos de apenas alguns componentes vulneráveis é mais adequado considerar o reparo que torna o sistema que falhou em um nível intermediário. Logo, as ações de reparo estão mais voltadas à manutenção do sistema como um todo do que propriamente ao sistema originador da falha.

Em 1983, Brown e Proschan investigaram o processo de falha, em que para cada tempo de falha ocorre um reparo perfeito com probabilidade $p$ e um reparo imperfeito ocorre com probabilidade $1-p$, independente da história prévia da falha (Brown e Proschan 1983).

Em 1989, a ideia de idade virtual de um sistema foi introduzida por Kijima (1989), que é uma função positiva de sua idade real e de seu histórico de falhas. O modelo de idade virtual proposto insere um parâmetro, denotado por $\theta(0 \leq \theta \leq 1)$, o qual representa o grau de eficiência dos reparos. Um sistema com idade virtual $\nu>0$ é assumido ter comportamento exatamente como um novo sistema o qual tem alcançado idade $\nu$ sem ter falhado.

E em 2004, duas classes de modelos para reparo imperfeito foram propostas por Doyen e Gaudoin (2004), a classe de modelo ARI - Arithmetic Reduction of Intensity e a classe de modelo ARA - Arithmetic Reduction of Age. Estas classes de modelos são definidas pela sua memória de ordem $m$, em que $m$ refere-se ao número máximo de falhas anteriores envolvidas no cálculo da função intensidade. Um caso particular é para $m=1$, que indica que cada ação de reparo reduz o desgaste do sistema ocorrido apenas desde a última falha. Isto é, a função intensidade do sistema é recalculada a cada falha, considerando o tempo da falha mais recente. Nesse estudo foi considerado este caso particular.

\section{Modelo ARA}

Neste modelo, o efeito do reparo é expresso por uma redução na idade virtual do sistema, e a sua função de intensidade é dada por

$$
\rho_{A R A}(t)=\lambda\left(t-(1-\theta) T_{N(t)}\right)
$$

Este modelo tem como casos especiais o reparo mínimo e o reparo perfeito. Quando $\theta=1$, o modelo iguala-se ao reparo mínimo, isto é:

$$
\rho_{A R A}(t)=\lambda\left(t-(1-1) T_{N(t)}\right)=\lambda\left(t-0 T_{N(t)}\right)=\lambda(t)
$$


então, vê-se que esta função é igual ao modelo de reparo mínimo dado pela função (2.7).

O outro caso especial ocorre quando $\theta=0$, em que o modelo fica sendo o de reparo perfeito, ou seja,

$$
\rho_{A R A}(t)=\lambda\left(t-(1-0) T_{N(t)}\right)=\lambda\left(t-T_{N(t)}\right)
$$

que é a função de intensidade de falhas do reparo perfeito (2.9).

Considerando o processo de lei de potência (2.5), a função de intensidade para o modelo ARA (2.11) passa a ser:

$$
\rho_{A R A}(t)=\frac{\beta}{\eta}\left(\frac{t-(1-\theta) T_{N(t)}}{\eta}\right)^{\beta-1} .
$$

\section{Modelo ARI}

No modelo ARI, cada ação de reparo reduz a função de intensidade de falha do sistema e entre duas falhas consecutivas sua intensidade de falha é verticalmente paralela à sua intensidade original. Assim, a função de intensidade de falha é expressa por:

$$
\rho_{A R I}(t)=\lambda(t)-(1-\theta) \lambda\left(T_{N(t)}\right)
$$

Considerando o PLP (2.5), a função de intensidade (2.13) do modelo ARI fica sendo

$$
\rho_{A R I}(t)=\frac{\beta}{\eta}\left(\frac{t}{\eta}\right)^{\beta-1}(1-\theta)\left[\frac{\beta}{\eta}\left(\frac{T_{N(t)}}{\eta}\right)^{\beta-1}\right] .
$$

A fim de ilustrar a diferença entre os modelos ARA e ARI, um gráfico de ambos modelos foi realizado supondo alguns valores fixados para os parâmetros destes modelos, em que $\beta=4, \eta=1, \theta=0.3$ e supôs-se também que as falhas foram observadas nos tempos $T_{1}=2$ e $T_{2}=3$.

Assim, a função de intensidade para o modelo ARA fica sendo:

$$
\rho_{A R A}=\left\{\begin{array}{l}
\lambda(t)=4 t^{3}, 0 \leq t<2 \\
\lambda(t-0,7 \times 2)=4(t-1,4)^{3}, 2 \leq t<3 \\
\lambda(t-0,7 \times 3)=3(t-2,1)^{3}, 3 \leq t<\ldots
\end{array}\right.
$$


E para o modelo ARI a função de intensidade é

$$
\rho_{A R I}=\left\{\begin{array}{l}
\lambda(t)=4 t^{3}, 0 \leq t<2 \\
\lambda(t)-0.7 \times \lambda(2)=4(t)^{3}-0,7 \times 4(2)^{3}, 2 \leq t<3 \\
\lambda(t)-0.7 \times \lambda(3)=4(t)^{2}-0,7 \times 3(3)^{2}, 3 \leq t<\ldots
\end{array}\right.
$$

Na figura (2.1) tem-se o esboço das funções de intensidade referente aos modelos ARA e ARI exemplificadas anteriormente, em que pode-se observar como a função de intensidade diminui após as falhas de cada modelo. No caso do modelo ARA, no tempo $T_{1}=2$, temse que a idade reduziu de 2 para 0,7 e no tempo $T_{2}=3$ a idade reduziu de 3 para, aproximadamente, 2,4. Para o modelo ARI, vê-se que no tempo $T_{1}=2$ a intensidade era de 32 e caí para 9,9 .
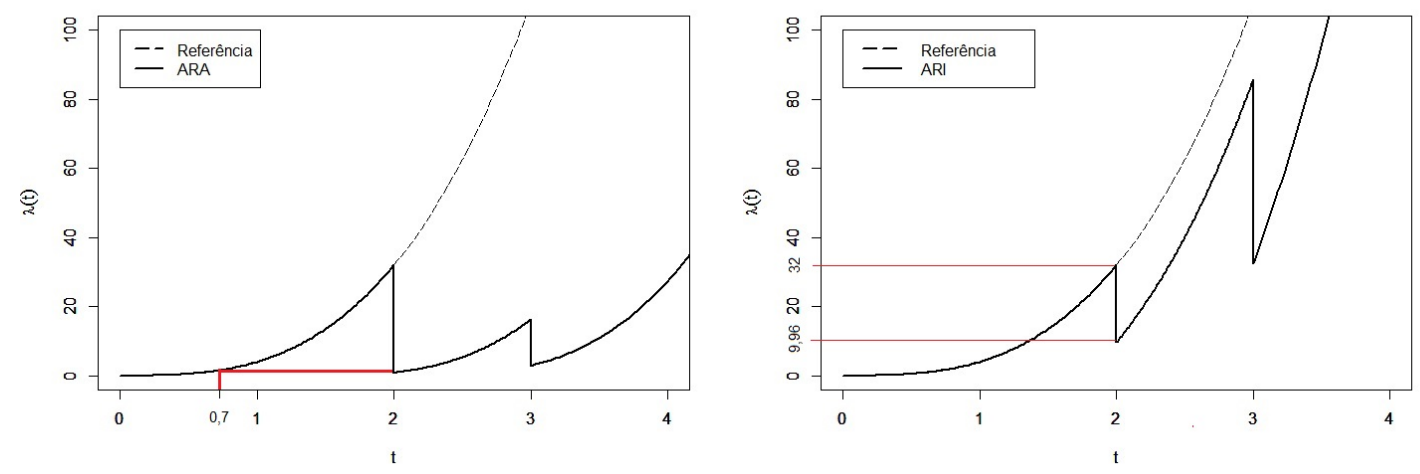

Figura 2.1: Funções de intensidade pelo PLP para modelos ARA e ARI.

Por meio da análise destes gráficos, pode-se ver que entre duas falhas consecutivas a função de intensidade do modelo ARA é horizontalmente paralela a idade original, enquanto que para o modelo ARI, entre duas falhas consecutivas a função de falha é verticalmente paralela à sua intensidade original.

\subsection{Inferência}

A fim de estimar os parâmetros dos modelos apresentados na seção anterior, o método considerado é o de máxima verossimilhança.

Primeiro será construída a função de verossimilhança para o modelo de reparo mínimo. Para tal, considere $k$ sistemas reparáveis, para $k=1,2, \ldots$, assumindo as 
seguintes condições:

- Seja $t_{i, j}$, para $i=(1,2, \ldots, k)$ e $j=\left(1,2, \ldots, n_{i}\right)$ observações da variável aleatória $T$ que representam os tempos de falha para o $i$-ésimo sistema, registrado como o tempo desde o início do sistema $\left(0<t_{i, 1}<t_{i, 2}<\ldots<t_{i, n_{i}}\right)$;

- O $i$-ésimo sistema truncado por tempo é observado até o tempo predeterminado $t_{i}^{*}$, sendo que $0<t_{i, 1}<t_{i, 2}<\ldots<t_{i, n_{i}}<t_{i}^{*}$ ou truncado em um número de falhas $n_{i}^{*}$ predeterminado, em que $\left(0<t_{i, 1}<t_{i, 2}<\ldots<t_{i, n_{i}^{*}}\right)$;

- $n_{i}$ falhas são observadas no $i$-ésimo sistema truncado por tempo ou por falha, $i=$ $1,2, \ldots, k$

- $N=\sum_{i=1}^{k} n_{i}$ é o número total de falhas observadas no estudo;

- Seja $\boldsymbol{\mu}=(\beta, \eta)$ o vetor de parâmetros a ser estimado.

A função de verossimilhança para este processo deve combinar a densidade de probabilidade conjunta dos tempos de falha dos $k$ sistemas. Conforme descrito no início deste capítulo, há basicamente duas maneiras de observar os dados de sistemas reparáveis, denominados por truncamento por tempo ou truncamento por falha. Considerando o caso de estudos truncados por tempo $\left(t_{i}^{*}\right)$, o número de falhas $(\mathrm{N}(\mathrm{t}))$ é aleatório e, portanto, este deve ser considerado na construção da função de verossimilhança, isto é

$$
\begin{aligned}
L\left(\boldsymbol{\mu} \mid t_{i}\right)= & \prod_{i=1}^{k} f\left(t_{i, 1}, t_{i, 2}, \ldots, t_{i, n_{i}}, n\right) \\
= & \prod_{i=1}^{k} f\left(t_{i, 1}\right) f\left(t_{i, 2} \mid t_{i, 1}\right) f\left(t_{i, 3} \mid t_{i, 1}, t_{i, 2}\right), \ldots, f\left(t_{i, n_{i}} \mid t_{i, 1}, t_{i, 2}, \ldots, t_{i, n_{i-1}}\right) \\
& \times P\left[N(t)=n \mid t_{i, 1}, t_{i, 2}, \ldots, t_{i, n_{i}}\right] .
\end{aligned}
$$

Para determinar a função $f\left(t_{1}\right)$, deve-se considerar que

$$
f\left(t_{1}\right)=\frac{d}{d t_{1}} F\left(t_{1}\right)=\frac{d}{d t_{1}}\left[1-R\left(t_{1}\right)\right]
$$

sendo que $\mathrm{R}(\mathrm{t})$ é a função de confiabilidade e para obtê-la, tem-se que a função $R\left(t_{1}\right)$ é dada por

$$
R\left(t_{1}\right)=P\left[T_{1}>t_{1}\right]=P\left[N\left(t_{1}\right)=0\right]=\mathrm{e}^{-\Lambda\left(t_{1}\right)} .
$$


Assim, a função (2.16) é dada por:

$$
f\left(t_{1}\right)=\lambda\left(t_{1}\right) \mathrm{e}^{-\Lambda\left(t_{1}\right)}
$$

Esta ideia é estendida para a obtenção das demais funções. Para determinar $f\left(t_{i, 2} \mid t_{i, 1}\right)$, tem-se que

$$
P\left[T_{2}>t_{2} \mid t_{1}\right]=P\left[N\left(t_{2}\right)-N\left(t_{1}\right)=0\right]=\mathrm{e}^{-\Lambda\left(t_{2}\right)+\Lambda\left(t_{1}\right)} .
$$

E então a função para $f\left(t_{i, 2} \mid t_{i, 1}\right)$ é dada por

$$
f\left(t_{2}\right)=\lambda\left(t_{2}\right) \mathrm{e}^{-\Lambda\left(t_{2}\right)+\Lambda\left(t_{1}\right)} .
$$

Como o reparo mínimo é um PPNH, os seus incrementos são independentes e, portanto, os tempos estarão condicionados apenas ao tempo imediatamente anterior.

Dessa forma, para obter a função $f\left(t_{i, 3} \mid t_{i, 1}, t_{i, 2}\right)$, tem-se que

$$
P\left[T_{2}>t_{3} \mid t_{2}\right]=P\left[N\left(t_{3}\right)-N\left(t_{2}\right)=0\right]=\mathrm{e}^{-\Lambda\left(t_{3}\right)+\Lambda\left(t_{2}\right)} .
$$

Logo,

$$
f\left(t_{3}\right)=\lambda\left(t_{3}\right) \mathrm{e}^{-\Lambda\left(t_{3}\right)+\Lambda\left(t_{2}\right)} .
$$

Para obter as demais funções, deve-se seguir esse raciocínio sucessivamente até $t_{n}$. Porém, ainda precisa-se calcular a parcela $P\left[N(t)=n \mid t_{i, 1}, t_{i, 2}, \ldots, t_{i, n_{i}}\right]$ e seguindo o raciocínio anterior, tem-se que

$$
P\left[N\left(t_{i}^{*}\right)=n \mid t_{n_{i}}\right]=P\left[N\left(t_{i}^{*}\right)-N\left(t_{n_{i}}\right)=0\right]=\mathrm{e}^{-\Lambda\left(t_{i}^{*}\right)+\Lambda\left(t_{n_{i}}\right)} .
$$

Agora, voltando a função (2.15), pode-se reescrevê-la como:

$L\left(\boldsymbol{\mu} \mid t_{i}\right)=\prod_{i=1}^{k} \lambda\left(t_{i, 1}\right) \mathrm{e}^{-\Lambda\left(t_{i, 1}\right)} \lambda\left(t_{i, 2}\right) \mathrm{e}^{-\Lambda\left(t_{i, 2}\right)+\Lambda\left(t_{i, 1}\right)} \ldots \lambda\left(t_{i, n_{i}}\right) \mathrm{e}^{-\Lambda\left(t_{i, n_{i}}\right)+\Lambda\left(t_{i, n_{i-1}}\right)} \mathrm{e}^{-\Lambda\left(t_{i}^{*}\right)+\Lambda\left(t_{n_{i}}\right)}$

Como os termos $\Lambda\left(t_{i, j}\right)$ se cancelam, para $j=2, \ldots n-1$, a função de verossimilhança 
para o modelo de reparo mínimo é, então, dada por:

$$
L\left(\boldsymbol{\mu} \mid t_{i}\right)=\prod_{i=1}^{k} \lambda\left(t_{i, 1}\right) \lambda\left(t_{i, 2}\right) \ldots \lambda\left(t_{i, n_{i}}\right) \mathrm{e}^{-\Lambda\left(t_{i}^{*}\right)}
$$

Ou ainda

$$
L\left(\boldsymbol{\mu} \mid t_{i, j}\right)=\prod_{i=1}^{k} \prod_{j=1}^{n_{i}}\left[\lambda\left(t_{i, j}\right)\right] \mathrm{e}^{-\Lambda\left(t_{i}^{*}\right)}
$$

Considerando a função (2.5) do PLP, a função de verossimilhança truncada por tempo para o RM é, então, dada por

$$
L_{R M}\left(\boldsymbol{\mu} \mid t_{i, j}\right)=\prod_{i=1}^{k} \prod_{j=1}^{n_{i}}\left[\beta \eta^{-\beta}\left(t_{i, j}\right)^{\beta-1}\right] \exp \left\{-\eta^{-\beta}\left(t_{i}^{*}\right)^{\beta}\right\}
$$

em que $t_{i}^{*}$ é o tempo predeterminado de truncamento para o $i$-ésimo sistema. Se o estudo foi truncado por falha, basta substituir $t_{i}^{*}$ por $t_{i, n_{i} *}$, que é o número de falhas truncadas predeterminadas para o $i$-ésimo sistema.

E o logaritmo da função de verossimilhança é dado por

$$
\begin{aligned}
\ell_{R M}\left(\boldsymbol{\mu} \mid t_{i, j}\right) & =\sum_{i=1}^{k} n_{i} \log (\beta)-\beta \sum_{i=1}^{k} n_{i} \log (\eta)+(\beta-1) \sum_{i=1}^{k} \sum_{j=1}^{n_{i}}\left(t_{i, j}\right)-\eta^{-\beta} \sum_{i=1}^{k}\left(t_{i}^{*}\right)^{\beta} \\
& =N \log (\beta)-\beta N \log (\eta)+(\beta-1) \sum_{i=1}^{k} \sum_{j=1}^{n_{i}}\left(t_{i, j}\right)-\eta^{-\beta} \sum_{i=1}^{k}\left(t_{i}^{*}\right)^{\beta}
\end{aligned}
$$

em que $N=\sum_{i=1}^{k} n_{i}$, que representa o total de falhas ocorridas no estudo.

Similarmente ao RM, para construir a função de verossimilhança para as classes de modelo ARA e ARI considere $k$ sistemas reparáveis, para $k=1,2, \ldots$, assumindo as seguintes condições:

- Para cada falha uma ação de reparo de grau $\theta$ é realizada, para $\theta=[0,1]$;

- Seja $t_{i, j}$, para $i=(1,2, \ldots, k)$ e $j=\left(1,2, \ldots, n_{i}\right)$ observações da variável aleatória $T$ que representam os tempos de falha para o $i$-ésimo sistema, registrado como o tempo desde o início do sistema $\left(0<t_{i, 1}<t_{i, 2}<\ldots<t_{i, n_{i}}\right)$;

- O $i$-ésimo sistema truncado por tempo é observado até o tempo predeterminado $t_{i}^{*}$, sendo que $0<t_{i, 1}<t_{i, 2}<\ldots<t_{i, n_{i}}<t_{i}^{*}$ ou truncado em um número de falhas $n_{i}^{*}$ 
predeterminado, em que $\left(0<t_{i, 1}<t_{i, 2}<\ldots<t_{i, n_{i}^{*}}\right)$;

- $n_{i}$ falhas são observadas no $i$-ésimo sistema truncado por tempo ou por falha, $i=$ $1,2, \ldots, k$

- $N=\sum_{i=1}^{k} n_{i}$ é o número total de falhas observadas no estudo;

- Seja $\boldsymbol{\mu}=(\beta, \eta, \theta)$ o vetor de parâmetros a ser estimado.

A função de verossimilhança para este processo deve combinar a densidade de probabilidade conjunta dos tempos de falha dos $k$ sistemas.

No caso de um estudo conduzido por um truncamento por falha, a função de verossimilhança é dada por

$$
\begin{aligned}
L\left(\boldsymbol{\mu} \mid t_{i}\right) & =\prod_{i=1}^{k} f\left(t_{i, 1}, t_{i, 2}, \ldots, t_{i, n_{i}^{*}}\right) \\
& =\prod_{i=1}^{k} f\left(t_{i, 1}\right) f\left(t_{i, 2} \mid t_{i, 1}\right) f\left(t_{i, 3} \mid t_{i, 1}, t_{i, 2}\right), \ldots, f\left(t_{i, n_{i}^{*}} \mid t_{i, 1}, t_{i, 2}, \ldots, t_{i, n_{i-1}}\right) .
\end{aligned}
$$

As funções podem ser escritas como $f\left(t_{i, j} \mid t_{i, j-1}\right)=\frac{d}{d t_{i, j}} F\left(t_{i, j} \mid t_{i, j-1}\right)=\frac{d}{d t_{i, j}}\left(1-R\left(t_{i, j} \mid t_{i, j-1}\right)\right)$, para $j=1, \ldots, n_{i}^{*}$. Assim, tem-se que

$$
\begin{aligned}
f\left(t_{i, 1}\right) & =\frac{d}{d t_{i, 1}}\left[1-R\left(t_{i, 1}\right)\right] \\
f\left(t_{i, 2}\right) & =\frac{d}{d t_{i, 2}}\left[1-R\left(t_{i, 2} \mid t_{i, 1}\right)\right] \\
f\left(t_{i, 3}\right) & =\frac{d}{d t_{i, 3}}\left[1-R\left(t_{i, 3} \mid t_{i, 1}, t_{i, 2}\right)\right] \\
\vdots & \\
f\left(t_{i, n_{i}^{*}}\right) & =\frac{d}{d t_{i, n_{i}^{*}}}\left[1-R\left(t_{i, n_{i}^{*}} \mid t_{i, 1}, t_{i, 2}, \ldots, t_{i, n_{i-1}}\right)\right] .
\end{aligned}
$$

Devido à propriedade de incrementos independentes, a função condicional irá depender 
apenas do tempo imediatamente anterior, portanto, as funções anteriores ficam

$$
\begin{aligned}
f\left(t_{i, 1}\right) & =\frac{d}{d t_{i, 1}}\left[1-R\left(t_{i, 1}\right)\right] \\
f\left(t_{i, 2}\right) & =\frac{d}{d t_{i, 2}}\left[1-R\left(t_{i, 2} \mid t_{i, 1}\right)\right] \\
f\left(t_{i, 3}\right) & =\frac{d}{d t_{i, 3}}\left[1-R\left(t_{i, 3} \mid t_{i, 2}\right)\right] \\
\vdots & \\
f\left(t_{i, n_{i}^{*}}\right) & =\frac{d}{d t_{i, n_{i}^{*}}}\left[1-R\left(t_{i, n_{i}^{*}} \mid t_{i, n_{i-1}}\right)\right]
\end{aligned}
$$

Para a construção da função de verossimilhança dos modelos ARA e ARI o raciocínio é o mesmo, mas, agora deve-se considerar a definição de suas funções de intensidade.

Assim sendo, para o modelo modelo ARA a função de confiabilidade condicional é dada por

$$
\begin{aligned}
R\left(t_{i, j} \mid t_{i, j-1}\right) & =P\left(T>t_{i, j} \mid t_{i, j-1}\right)=P\left(N\left(t_{i, j}, t_{i, j-1}\right]=0\right) \\
& =\exp \left(-\Lambda\left(t_{i, j}-(1-\theta) t_{i, j-1}\right)+\Lambda\left(\theta t_{i, j-1}\right)\right) .
\end{aligned}
$$

E desta forma, calculando as derivadas da função (2.22), tem-se que a função de verossimilhança do modelo ARA é estabelecida por

$$
\begin{aligned}
& L\left(\boldsymbol{\mu} \mid t_{i, j}\right)=\prod_{i=1}^{k} \prod_{j=1}^{n_{i}^{*}} \lambda\left(t_{i, j}-(1-\theta) T_{N\left(t_{i, j}\right)}\right) \exp \left(-\Lambda\left(t_{i, j}-(1-\theta) T_{N\left(t_{i, j}\right)}\right)+\Lambda\left(\theta t_{i, j-1}\right)\right) \\
& \text { para } i=1, \ldots, k \text { e } j=1, \ldots, n_{i} .
\end{aligned}
$$

Pode-se reescrever o último termo da seguinte maneira: $\exp \left(\Lambda\left(\theta t_{i, j-1}\right)\right)=\exp \left(\Lambda\left(\theta t_{i, j}\right)-\right.$ $\left.\Lambda\left(\theta t_{i, n_{i}^{*}}\right)\right)$. Logo, a função (2.23) fica sendo

$$
\begin{aligned}
L\left(\boldsymbol{\mu} \mid t_{i, j}\right)= & \prod_{i=1}^{k} \prod_{j=1}^{n_{i}^{*}}\left[\lambda\left(t_{i, j}-(1-\theta) T_{N\left(t_{i, j}\right)}\right) \exp \left(-\Lambda\left(t_{i, j}-(1-\theta) T_{N\left(t_{i, j}\right)}\right)+\Lambda\left(\theta t_{i, j}\right)\right)\right] \\
& \exp \left(-\Lambda\left(t_{i, j}-(1-\theta) T_{N\left(t_{i, j}\right)}\right)+\Lambda\left(\theta t_{i, j}\right)\right)
\end{aligned}
$$


Sob o PLP, a função de verossimilhança para o modelo ARA é, então, definida por:

$$
\begin{aligned}
L_{A R A}(\boldsymbol{\mu})= & \prod_{i=1}^{k} \prod_{j=1}^{n_{i}^{*}}\left\{\left[\frac{\beta}{\eta}\left(\frac{t_{i, j}-(1-\theta) T_{N\left(t_{i, j}\right)}}{\eta}\right)^{\beta-1}\right] \exp \left[\left(\frac{\theta t_{i, j}}{\eta}\right)^{\beta}-\left(\frac{t_{i, j}-(1-\theta) T_{N\left(t_{i, j}\right)}}{\eta}\right)^{\beta}\right]\right\} \\
& \exp \left[-\left(\frac{\theta t_{i, n_{i}^{*}}}{\eta}\right)^{\beta}\right]
\end{aligned}
$$

Agora, seguindo o mesmo raciocínio anterior e considerando a sua função de intensidade (2.13), a função de verossimilhança para o modelo ARI é dada por:

$$
\begin{aligned}
L\left(\boldsymbol{\mu} \mid t_{i, j}\right)= & \prod_{i=1}^{k} \prod_{j=1}^{n_{i}^{*}} \lambda\left(t_{i, j}\right)-(1-\theta) \lambda\left(T_{N\left(t_{i, j}\right)}\right) \exp \left(-\Lambda\left(t_{i, j}\right)+\Lambda\left(T_{N\left(t_{i, j}\right)}\right)\right) \\
& \left.\exp \left((1-\theta) \Lambda\left(T_{N\left(t_{i, j}\right)}\right)\left[t_{i, j}-T_{N\left(t_{i, j}\right)}\right)\right]\right)
\end{aligned}
$$

Sob PLP, a função (2.26) é estabelecida por

$$
\begin{aligned}
L_{A R I}(\boldsymbol{\mu})= & \prod_{i=1}^{k} \prod_{j=1}^{n_{i}} \frac{\beta}{\eta}\left[\left(\frac{t_{i, j}}{\eta}\right)^{\beta-1}-(1-\theta)\left(\frac{T_{N\left(t_{i, j}\right)}}{\eta}\right)^{\beta-1}\right] \\
& \exp \left\{-\left(\frac{t_{i, j}}{\eta}\right)^{\beta}+\left(\frac{T_{N\left(t_{i, j}\right)}}{\eta}\right)^{\beta}+(1-\theta) \frac{\beta}{\eta}\left(\frac{T_{N\left(t_{i, j}\right)}}{\eta}\right)^{\beta-1}\left(t_{i, j}-T_{N\left(t_{i, j}\right)}\right)\right\} .
\end{aligned}
$$

Quando o sistema é observado até um tempo predeterminado, caracterizando o truncamento por tempo, o número de falhas $N(t)$ é uma variável aleatória e ela deve ser levada em conta na função de verossimilhança. Assim, tal função fica sendo

$$
\begin{aligned}
L\left(\boldsymbol{\mu} \mid t_{i, j}\right) & =\prod_{i=1}^{k} f\left(t_{i, 1}, t_{i, 2}, \ldots, t_{i, n_{i}} \mid n_{i}\right) P\left(N\left(t_{i}^{*}\right]=n_{i}\right) \\
& =\prod_{i=1}^{k} \lim _{h \rightarrow 0} \frac{P\left(t_{i, 1}-h<T_{i, 1} \leq t_{i, 1}, \ldots, t_{i, n_{i}}-h<T_{i, n_{i}} \leq t_{i, n_{i}} \mid N\left(t_{i}^{*}\right]=n_{i}\right)}{h^{n_{i}}} P\left(N\left(t_{i}^{*}\right]=n_{i}\right) \\
& =\prod_{i=1}^{k} \lim _{h \rightarrow 0} \frac{P\left(t_{i, 1}-h<T_{i, 1} \leq t_{i, 1}, \ldots, t_{i, n_{i}}-h<T_{i, n_{i}} \leq t_{i, n_{i}}, N\left(t_{i}^{*}\right]=n_{i}\right) P\left(N\left(t_{i}^{*}\right]=n_{i}\right)}{h^{n_{i}} P\left(N\left(t_{i}^{*}\right]=n_{i}\right)} \\
& =\prod_{i=1}^{k} \lim _{h \rightarrow 0} \frac{P\left(t_{i, 1}-h<T_{i, 1} \leq t_{i, 1}, \ldots, t_{i, n_{i}}-h<T_{i, n_{i}} \leq t_{i, n_{i}}, N\left(t_{i}^{*}\right]=n_{i}\right)}{h^{n_{i}}}
\end{aligned}
$$




$$
\begin{aligned}
& =\prod_{i=1}^{k} \lim _{h \rightarrow 0} \frac{P\left(N\left(0, t_{i, 1}-h\right]=0\right) P\left(N\left(t_{i, 1}-h, t_{i, 1}\right]=1\right) \ldots P\left(N\left(t_{i, n_{i}}-h, t_{i, n_{i}}\right]=1\right) P\left(N\left(t_{i, n_{i}}, t_{i}^{*}\right]=0\right)}{h^{n_{i}}} \\
& =\prod_{i=1}^{k} \lim _{h \rightarrow 0}\left[\frac{\prod_{j=1}^{n_{i}}\left\{P\left(N\left(t_{i, j-1}, t_{i, j}-h\right]=0\right) P\left(N\left(t_{i, j}-h, t_{i, j}\right]=1\right)\right\} P\left(N\left(t_{i, n_{i}}, t_{i}^{*}\right]=0\right)}{h^{n_{i}}}\right] .
\end{aligned}
$$

Tem-se que

$$
E\left(N\left(t_{i, j-1}, t_{i, j}-h\right]\right)=\int_{t_{i, j-1}}^{t_{i, j}-h} \lambda(x) d x=\Lambda\left(t_{i, j}-h\right)-\Lambda\left(t_{i, j-1}\right) .
$$

E tem-se também que

$P\left(N\left(t_{i, j-1}, t_{i, j}-h\right]=0\right)=\exp \left(-E\left(N\left(t_{i, j-1}, t_{i, j}-h\right]\right)\right)=\exp \left(-\left(\Lambda\left(t_{i, j}-h\right)-\Lambda\left(t_{i, j-1}\right)\right)\right)$

e

$$
\begin{aligned}
P\left(N\left(t_{i, j}-h, t_{i, j}\right]=1\right) & =E\left(N\left(t_{i, j}-h, t_{i, j}\right]\right) \exp \left(-E\left(N\left(t_{i, j}-h, t_{i, j}\right]\right)\right) \\
& =\Lambda\left(t_{i, j}\right)-\Lambda\left(t_{i, j}-h\right) \exp \left(-\left(\Lambda\left(t_{i, j}\right)-\Lambda\left(t_{i, j}-h\right)\right)\right) .
\end{aligned}
$$

Agora, voltando à equação (2.28), tem-se que

$L\left(\boldsymbol{\mu} \mid t_{i, j}\right)=\prod_{i=1}^{k} \lim _{h \rightarrow 0}\left[\frac{\prod_{j=1}^{n_{i}} \Lambda\left(t_{i, j}\right)-\Lambda\left(t_{i, j}-h\right) \exp \left\{-\left(\Lambda\left(t_{i, j}\right)-\Lambda\left(t_{i, j-1}\right)\right)\right\} \exp \left(-\left(\Lambda\left(t_{i}^{*}\right)-\Lambda\left(t_{i, j-1}\right)\right)\right)}{h^{n_{i}}}\right]$

E então

$L\left(\boldsymbol{\mu} \mid t_{i, j}\right)=\prod_{i=1}^{k} \lim _{h \rightarrow 0} \prod_{j=1}^{n_{i}}\left(\left[\frac{\Lambda\left(t_{i, j}\right)-\Lambda\left(t_{i, j}-h\right)}{h^{n_{i}}}\right] \exp \left\{-\Lambda\left(t_{i, j}\right)+\Lambda\left(t_{i, j-1}\right)\right\}\right) \exp \left(-\Lambda\left(t_{i}^{*}\right)+\Lambda\left(t_{i, j-1}\right)\{2.30)\right.$

O resultado desta função (2.30) tendo em vista a função de intensidade (2.11), gera a função de verossimilhança para o modelo ARA, que é dada por

$$
\begin{aligned}
L_{A R A}(\boldsymbol{\mu})= & \prod_{i=1}^{k} \prod_{j=1}^{n_{i}}\left[\lambda\left(t_{i, j}-(1-\theta) T_{N\left(t_{i, j}\right)}\right) \exp \left(-\Lambda\left(t_{i, j}-(1-\theta) T_{N\left(t_{i, j}\right)}\right)+\Lambda\left(\theta t_{i, j}\right)\right]\right. \\
& \exp \left\{-\Lambda\left(t_{i}^{*}-(1-\theta) t_{i, n_{i}}\right)\right\} .
\end{aligned}
$$


E sob PLP a função (2.31) é determinada da seguinte maneira

$$
\begin{aligned}
L_{A R A}(\boldsymbol{\mu})= & \prod_{i=1}^{k} \prod_{j=1}^{n_{i}}\left\{\left[\frac{\beta}{\eta}\left(\frac{t_{i, j}-(1-\theta) T_{N\left(t_{i, j}\right)}}{\eta}\right)^{\beta-1}\right] \exp \left[\left(\frac{\theta t_{i, j}}{\eta}\right)^{\beta}-\left(\frac{t_{i, j}-(1-\theta) T_{N\left(t_{i, j}\right)}}{\eta}\right)^{\beta}\right]\right. \\
& \left.\exp \left[-\left(\frac{t_{i}^{*}-(1-\theta) t_{i, n_{i}}}{\eta}\right)^{\beta}\right]\right\} .
\end{aligned}
$$

O resultado da função (2.30) considerando a função (2.13) permite determinar a função de verossimilhança para o modelo ARI, que, sob PLP é dada por

$$
\begin{aligned}
L_{A R I}(\boldsymbol{\mu})= & \prod_{i=1}^{k} \prod_{j=1}^{n_{i}}\left\{\frac{\beta}{\eta}\left[\left(\frac{t_{i, j}}{\eta}\right)^{\beta-1}-(1-\theta)\left(\frac{T_{N\left(t_{i, j}\right)}}{\eta}\right)^{\beta-1}\right]\right. \\
& \left.\exp \left(-\left(\frac{t_{i, j}}{\eta}\right)^{\beta}+\left(\frac{T_{N\left(t_{i, j}\right)}}{\eta}\right)^{\beta}+(1-\theta) \frac{\beta}{\eta}\left(\frac{T_{N\left(t_{i, j}\right)}}{\eta}\right)^{\beta-1}\left(t_{i, j}-T_{N\left(t_{i, j}\right)}\right)\right)\right\} \\
& \exp \left(-\left(\frac{t_{i}^{*}}{\eta}\right)^{\beta}-\left(\frac{t_{i, n_{i}}}{\eta}\right)^{\beta}+(1-\theta) \frac{\beta}{\eta}\left(\frac{t_{i, n_{i}}}{\eta}\right)^{\beta-1}\left(t_{i}^{*}-t_{i, n_{i}}\right)\right) .
\end{aligned}
$$

O logaritmo das funções (2.32) e (2.34) são dadas respectivamente por:

$$
\begin{aligned}
\ell_{A R A}(\boldsymbol{\mu})= & N \log (\beta)-\beta N \log (\eta)+(\beta-1) \sum_{i=1}^{k} \sum_{j=1}^{n_{i}} \log \left[t_{i, j}-(1-\theta) T_{N\left(t_{i, j}\right)}\right] \\
& -\eta^{-\beta} \sum_{i=1}^{k} \sum_{j=1}^{n_{i}}\left(t_{i, j}-(1-\theta) T_{N\left(t_{i, j}\right)}\right)^{\beta}+-\eta^{-\beta} \sum_{i=1}^{k} \sum_{j=1}^{n_{i}}\left(\theta T_{N\left(t_{i, j}\right)}\right)^{\beta} \\
& -\eta^{-\beta} \sum_{i=1}^{k}\left(t_{i}^{*}-(1-\theta) t_{i, n_{i}}\right)^{\beta}
\end{aligned}
$$

e

$$
\begin{aligned}
\ell_{A R I}(\boldsymbol{\mu})= & N \log (\beta)-\beta N \log (\eta)+\sum_{i=1}^{k} \sum_{j=1}^{n_{i}} \log \left[\left(t_{i, j}\right)^{\beta-1}-(1-\theta)\left(T_{N\left(t_{i, j}\right)}\right)^{\beta-1}\right] \\
& -\eta^{-\beta} \sum_{i=1}^{k} \sum_{j=1}^{n_{i}}\left[\left(t_{i, j}\right)^{\beta}-\left(T_{N\left(t_{i, j}\right)}\right)^{\beta}-\beta(1-\theta)\left(T_{N\left(t_{i, j}\right)}\right)^{\beta-1}\left(t_{i, j}-T_{N\left(t_{i, j}\right)}\right)\right] \\
& -\eta^{-\beta} \sum_{i=1}^{k}\left[\left(t_{i}^{*}\right)^{\beta}-t_{i, n_{i}}^{\beta}-\beta(1-\theta) t_{i, n_{i}}^{\beta-1}\left(t_{i}^{*}-t_{i, n_{i}}\right)\right] .
\end{aligned}
$$

Como não é possível obter solução analítica, os métodos numéricos são considerados para estimação dos parâmetros. 
Vale ressaltar que, se em um mesmo estudo houver os dois tipos de truncamento, basta multiplicar a função de verossimilhança do truncamento por falha com a função de verossimilhança do truncamento por tempo dos respectivos modelos.

\subsection{Estudo de Simulação para o modelo ARA}

A fim de verificar as propriedades frequentistas, isto é, verificar se o procedimento assintótico é válido para pequenos e moderados tamanhos de amostra, um estudo de simulação foi realizado para a classe de modelos ARA. Para iniciar o processo de simulação, primeiramente os parâmetros foram fixados como segue: $\beta=1,33, \eta=4,94$ e $\theta=0,02$, considerando um tipo de estudo truncado por falha, que neste caso foi considerado que os $k$ sistemas teriam 3 falhas.

Utilizou-se três tamanhos amostrais, que neste caso são o número de sistemas: $k=50$, $k=100$ e $k=200$ e foram geradas $d=1.000$ réplicas. A fim de otimizar o processo de simulação, os valores iniciais utilizados foram os mesmos valores pré-fixados para os parâmetros. Para atender os objetivos do estudo de simulação, utilizou-se o seguinte algoritmo:

1. Fixar os valores dos parâmetros;

2. Gerar $u_{i} \sim U(0,1)$;

3. Fazer $T_{N\left(t_{i, 1}\right)}=0$

4. Gerar $t_{i, j}$, tal que $t_{i, j}=\left(-\log \left(1-u_{i}\right) \eta^{\beta}+\left(\theta T_{N\left(t_{i, j}\right)}\right)^{\beta}\right)^{\left(\frac{1}{\beta}\right)}+(1-\theta) T_{N\left(t_{i, j}\right)}$, com $\left.T_{N\left(t_{i, j}\right)}\right)=t_{i, j-1}$

5. Repetir os passos do item 2 ao item 4 por $k$ vezes;

6. Repetir os passos do item 2 a 5 até obter $d$ réplicas.

Para cada réplica da simulação foram obtidos o Erro Quadrático Médio (EQM) de cada estimativa, o Erro Padrão (EP), a amplitude e o intervalo de confiança de 95\%, baseado na teoria assintótica.

O EQM foi calculado da seguinte maneira:

$$
E Q M=\sum_{i=1}^{d} \frac{\left(\mu-\widehat{\mu}_{i}\right)^{2}}{d}
$$


em que $\mu$ representa um dos três parâmetros previamente fixados e $\widehat{\mu}$ representa a estimativa dos respectivos parâmetros na $i$-ésima réplica, cujo número de réplicas é $d=1000$.

O intervalo com $95 \%$ de confiança $(\mathrm{IC}(95 \%))$ foi calculado da seguinte forma:

$$
I C(1-\phi)=\widehat{\mu_{i}} \pm \sigma_{i} Z_{\phi / 2}
$$

em que $Z_{\phi / 2}$ é o quantil $\phi / 2$ da distribuição Normal e $\sigma_{i}$ é o erro padrão estimado para cada parâmetro $i$. O valor de $\phi$ utilizado foi de 0,05 .

Os resultados obtidos estão resumidos na Tabela (2.1) e na Figura (2.2), em que EMV são as estimativas obtidas via máxima verossimilhança. Os resultados mostram que conforme o tamanho da amostra aumenta, a amplitude do intervalo de confiança diminui para os três parâmetros do modelo. O erro quadrático médio cresce suavemente quando passa do tamanho amostra 50 para 100 e depois diminui consideravelmente para o tamanho de amostra maior, que é o tamanho de amostra 200. A média dos desvios diminuem conforme a amostra aumenta e a média das EMV se aproximam dos valores fixados, resultados de acordo com o esperado em um estudo de simulação para estimadores de máxima verossimilhança. 
Tabela 2.1: Resultados do estudo de simulação para o Modelo ARA

\begin{tabular}{ccccccc}
\hline Amostras & Parâmetros & EMV & EP & EQM & IC $(95 \%)$ & Amplitude \\
\hline $\mathrm{k}=50$ & $\hat{\beta}$ & 1,3668 & 0,1035 & 0,0119 & {$[1,163 ; 1,569]$} & 0,4057 \\
& $\hat{\eta}$ & 5,1007 & 0,4186 & 0,1909 & {$[4,280 ; 5,921]$} & 1,6410 \\
& $\hat{\theta}$ & 0,1110 & 0,1212 & 0,0278 & {$[0,000 ; 0,348]$} & 0,4750 \\
\hline $\mathrm{k}=100$ & $\hat{\beta}$ & 1,3488 & 0,0718 & 0,0055 & {$[1,207 ; 1,489]$} & 0,2816 \\
& $\hat{\eta}$ & 5,0279 & 0,2886 & 0,0831 & {$[4,462 ; 5,593]$} & 1,1313 \\
& $\hat{\theta}$ & 0,0806 & 0,0666 & 0,0079 & {$[0,049 ; 0,211]$} & 0,2612 \\
\hline $\mathrm{k}=200$ & $\hat{\beta}$ & 1,3433 & 0,0504 & 0,0026 & {$[1,244 ; 1,442]$} & 0,1975 \\
& $\hat{\eta}$ & 5,0231 & 0,2021 & 0,0458 & {$[4,626 ; 5,419]$} & 0,7922 \\
& $\hat{\theta}$ & 0,0716 & 0,0437 & 0,0044 & {$[0,000 ; 0,157]$} & 0,1714 \\
\hline
\end{tabular}

\subsection{Aplicação em dados reais}

Nesta seção é feita a aplicação aos dados dos cinco caminhões basculantes mencionado na seção (1.1). Toledo (2014) trabalhou com este conjunto de dados com o objetivo de verificar qual modelo ajustava-se melhor aos dados, em que foram considerados os modelos ARA e ARI com memória $m=1, \ldots, \infty$ e constatou que o melhor modelo era aquele com memória $m>10$. Como já foi mencionado, nesse trabalho a memória considerada é de $m=1$, em que cada ação de reparo reduz o desgaste do sistema ocorrido apenas desde a última falha.Assim, o intuito aqui não é identificar o modelo que melhor se ajusta aos dados, mas sim mostrar a aplicabilidade dos modelos.

Relembrando que os dados são provenientes de mineradora brasileira referente aos tempos de falha dos caminhões que operavam no transporte de minério do local de extração até o local de processamento. Os dados referem-se à cinco destes caminhões, cujos tempos de falhas foram computados em dias, no qual foram registradas 129 falhas no total.

Um esboço dos dados segue na Tabela 2.2.

Tabela 2.2: Esboço dos tempos de falha dos caminhões basculantes

\begin{tabular}{c|cccccccccc}
\hline Unidade & \multicolumn{8}{|c}{ Tempos de Falha em dias } \\
\hline 1 & 9 & 15 & 25 & $\ldots$ & 94 & 98 & 106 & & \\
2 & 3 & 5 & 11 & $\ldots$ & 66 & 69 & 72 & $\ldots$ & 100 & 103 \\
$\vdots$ & & & & $\vdots$ & & & & & & \\
5 & 3 & 9 & 26 & $\ldots$ & 91 & 93 & 99 & & & \\
\hline
\end{tabular}

Fonte: Toledo (2014) 

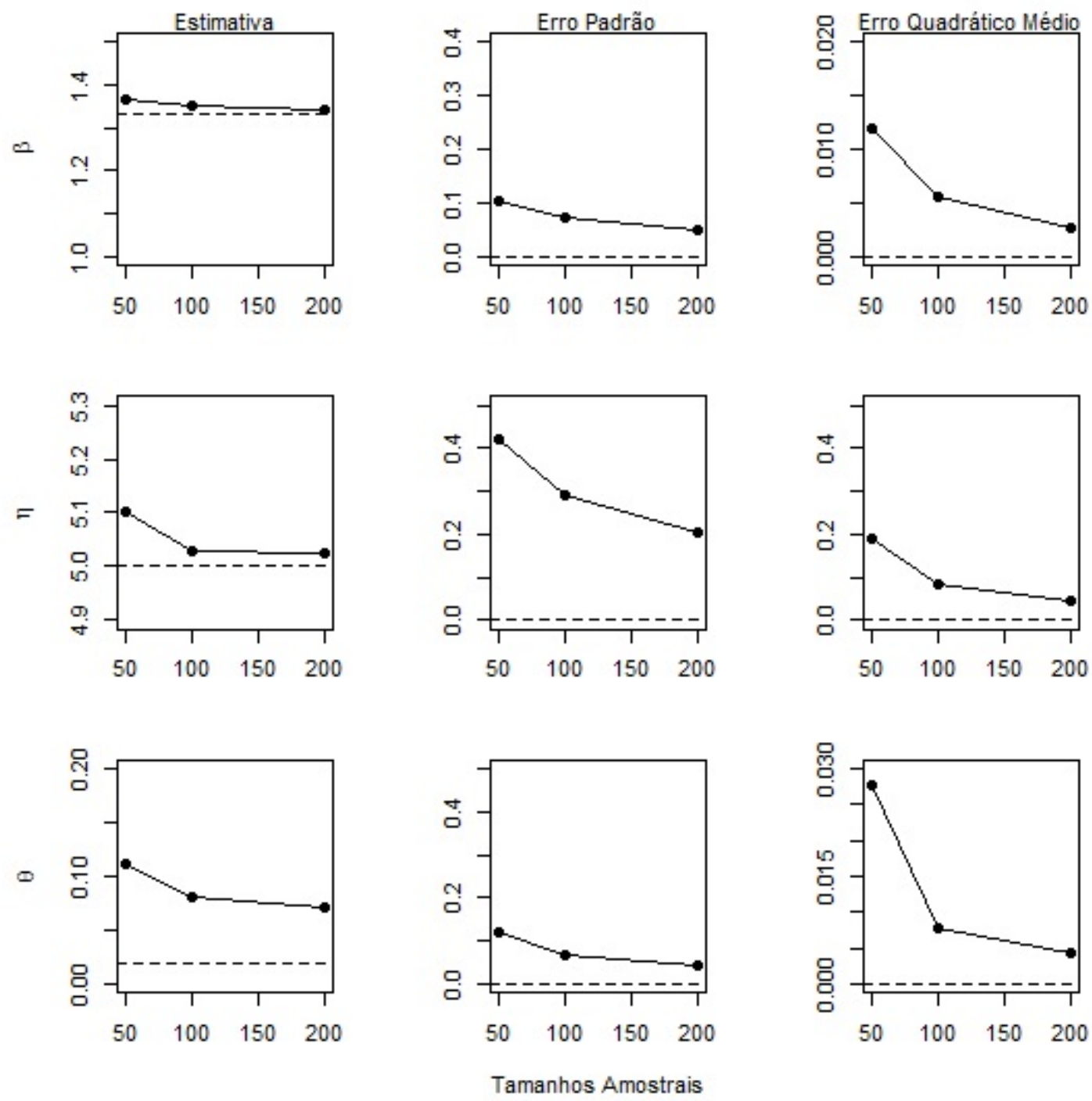

Figura 2.2: Gráfico dos Resultados da Simulação para o Modelo ARA

O software $\mathrm{R}$ foi utilizado para obter as estimativas pelo método de máxima verossimilhança para os modelos em questão, a partir da rotina optim pelo método de estimação default, que é o método simplex (Nelder e Mead 1965).

Conforme visto na Seção (2.2.1), para o modelo de RM, o parâmetro $\beta$ representa a elasticidade do número esperado de falhas em relação ao tempo, ou seja, quando $\beta>1$, indica que o sistema está deteriorando.

Para as classes de modelos ARA e ARI, o parâmetro $\theta$ representa o grau do efeito do reparo, se estiver próximo de zero, há indícios de que o reparo é perfeito e se estiver próximo de um, há indícios de reparo mínimo.

E o parâmetro $\eta$ pode ser interpretado como o tempo durante o qual se espera que exatamente uma falha ocorra. 
De acordo com as estimativas dispostas na Tabela (2.3), pode-se dizer que o sistema está deteriorando, pois a estimativa do parâmetro $\beta$ é maior que um para o modelo de RM e pode-se dizer que o tempo esperado para que ocorra uma falha é de, aproximadamente, 6 dias considerando o modelo de RM, de 5 dias pelo modelo ARA e 4 dias pelo modelo ARI. Pelo modelo ARA o efeito do reparo dá indícios de reparo perfeito, pois a estimativa do parâmetro $\theta$ é de 0,023 e pelo modelo ARI, há indicativo de reparo imperfeito $(0,232)$.

Tabela 2.3: Estimativas obtidas pelo Método Clássico de Máxima Verossimilhança

\begin{tabular}{ccccccc}
\hline & \multicolumn{3}{c}{ RM } & \multicolumn{2}{c}{ ARA } & \multicolumn{2}{c}{ ARI } \\
Parâmetros & EMV & IC $(0,95)$ & EMV & IC $(0,95)$ & EMV & IC $(0,95)$ \\
\hline$\hat{\beta}$ & 1,1361 & {$[0,940 ; 1,332]$} & 1,327 & {$[1,010 ; 1,644]$} & 1,4198 & {$[1,005 ; 1,833]$} \\
$\hat{\eta}$ & 5,9211 & {$[2,860 ; 8,973]$} & 4,928 & {$[3,109 ; 6,746]$} & 4,1834 & {$[2,152 ; 6,214]$} \\
$\hat{\theta}$ & - & - & 0,023 & {$[0,000 ; 0,097]$} & 0,2328 & {$[0,000 ; 0,601]$} \\
\hline
\end{tabular}

A qualidade do ajuste para o modelo de reparo mínimo foi avaliada comparando as estimativas da função de confiabilidade com a empírica de Kaplan-Meier (KM) para eventos recorrentes. Existem diferentes estimadores da função de confiabilidade, mas neste trabalho foi utilizado o estimador de Wang (Wang e Chang 1999) pois é um estimador para dados de tempos recorrentes correlacionados ou independentes e identicamente distribuídos. Sua função é derivada de um tipo estimador de Kaplan-Meier para eventos recorrentes com base nos intervalos entre os tempos, isto é $t_{i, j-1}-t_{i, j}$, os chamados gap times. Considere $T_{i j}$ o tempo entre o $j$-1-ésimo e $j$-ésimo evento do sistema $i, C_{i}$ o tempo de "censuras", isto é, o tempo entre o evento inicial e o final do acompanhamento do sistema $i$ e $m_{i}$ o índice tal que

$$
\sum_{j=1}^{m_{i}-1} T_{i j} \leq C_{i}
$$

e

$$
\sum_{j=1}^{m_{i}} T_{i j}>C_{i} .
$$

Dessa forma, o termo dados "não censurados" refere-se ao conjunto $\left(t_{i 1} t_{i 2}, \ldots, t_{i, m_{i}}\right)$ e o termo "dados censurados" refere-se ao conjunto $\left(t_{i 1} t_{i 2}, \ldots, t_{i, m_{i}}^{+}\right)$, em que $t_{i, m_{i}}^{+}$refere-se ao tempo entre o $m_{i}$-1-ésimo evento e o final do acompanhamento. Assim, $m_{i}$ é o número de eventos recorrentes para o sistema $i$. 
Considere também $m_{i}^{*}=1$ se $m_{i}=1$ e $m_{i}^{*}=m_{i}-1$ se $m_{i} \geq 2, y_{i j}$ os tempos de recorrências observados definido por $y_{i j}=t_{i j}$ se $j=1,2, \ldots, m_{i}-1$ e $y_{i j}=t_{i, m_{i}}^{+}$se $j=m_{i}$ e $S^{*}(t)$ o conjunto total de sistemas em risco em $t$, dado por

$$
S^{*}(t)=\sum_{i=1}^{n}\left[\frac{a_{i}}{m_{i}^{*}} \sum_{j=1}^{m_{i}^{*}} I\left(y_{i j} \geq t\right)\right]
$$

em que $a_{i}$ é uma função a valores positivos dos sistemas com valores censurados em que $E\left(a_{i}^{2}\right)<\infty$ e $I\left(y_{i j} \geq t\right)$ vale 0 se $y_{i j}<t$ e 1 se $y_{i j} \geq t$.

Seja

$$
d^{*}(t)=\sum_{i=1}^{n}\left[\frac{a_{i} I\left(m_{i} \geq 2\right)}{m_{i}^{*}} \sum_{j=1}^{m_{i}^{*}} I\left(y_{i j}=t\right)\right]
$$

Dessa forma, sejam $y_{1}^{*} y_{2}^{*}, \ldots, y_{1}^{K}$ os tempos "não censurados", distintos e ordenados, o estimador de Kaplan-Meier de Wang Chang é dado por

$$
\hat{R}(t)=\prod_{y_{i}^{*} \geq t}\left\{1-\frac{d^{*}\left(y_{i}^{*}\right)}{R^{*}\left(y_{i}^{*}\right)}\right\} .
$$

Esta fórmula exprime a função de confiabilidade do tempo entre dois eventos sucessivos, denominado como função de confiabilidade recorrente. Assume-se que os sistemas têm função de confiabilidade marginal comum e, às vezes, dentro de unidades interecorrentes elas estão correlacionados.

A Figura (2.3) apresenta o gráfico de estimativas de Kaplan-Meier para dados de eventos recorrentes versus estimativas de função de confiabilidade do modelo de RM. Nota-se que o decaimento da confiabilidade estimada segue a curva de Kaplan-Meier, sugerindo que o ajuste do modelo é adequado aos dados. 


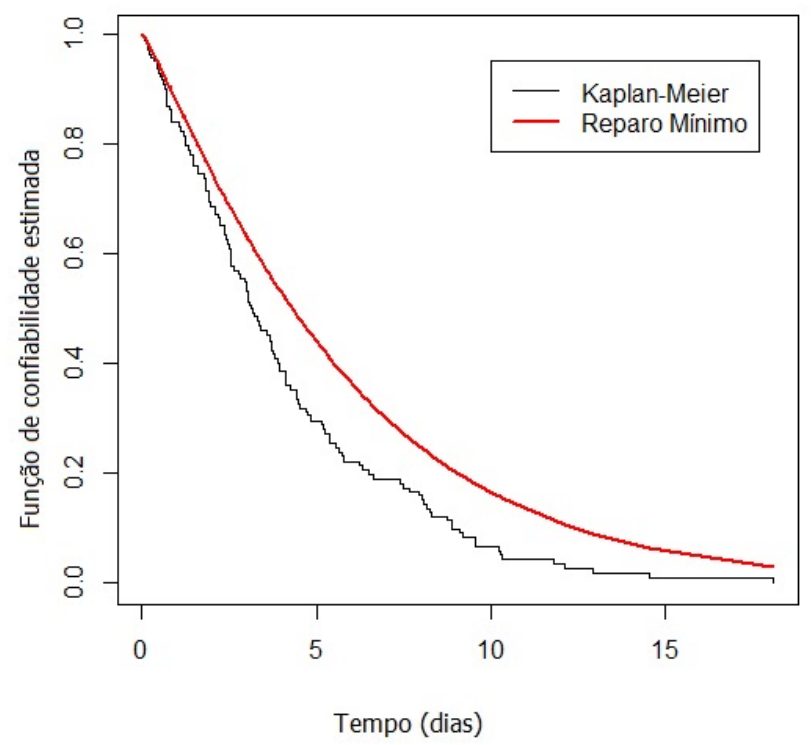

Figura 2.3: Gráfico de comparação de KM com função de confiabilidade estimada

\subsection{Alguns comentários}

Neste capítulo foram apresentadas as principais diferenças entre sistemas não reparáveis e sistemas reparáveis, bem como seus modelos usuais. Foram apresentados alguns conceitos básicos de processo de contagem necessários para o entendimento do modelo de reparo mínimo. O capítulo aborda também a ideia de idade virtual do sistema e as classes de modelos ARA e ARI, bem como o método de estimação clássico via máxima verossimilhança.

No estudo de simulação feito para o modelo ARA viu-se que a propriedade frequentista do erro quadrático médio foi atendida, conforme esperado para estimadores de máxima verossimilhança e na aplicação feita para um conjunto de dados reais, viu-se a aplicabilidade dos modelos, em que foi possível apontar a deterioração dos caminhões, seu tempo médio esperado para da ocorrência de uma falha e o grau de efeito do reparo.

No conjunto de dados analisado, viu-se que componentes do mesmo tipo foram estudados e nestes casos, acredita-se que é relevante verificar a heterogeneidade não observada entre estes caminhões. A partir dos modelos de fragilidade, tal heterogeneidade pode ser estudada e dentre os modelos usuais de sistemas reparáveis, o modelo de reparo mínimo será considerado para tal análise. Este modelo é apresentado no Capítulo 3, a seguir. 


\section{Capítulo 3}

\section{Modelo de Reparo Mínimo com fragilidade}

Os métodos estatísticos pressupõem que os tempos de falha de distintos sistemas são independentes. Em muitos estudos tal suposição é válida, entretanto, para outros ela pode ser inadequada (Colosimo e Giolo 2006). Uma situação em que a suposição de independência enttre os tempos de falha pode não ser válida ocorre quando os sistemas estão sujeitos a múltiplas ocorrências do mesmo evento, como é o caso dos dados referentes aos caminhões basculantes (Tabela 2.2), por isso é de interesse verificar se há heterogeneidade não observada entre sistemas deste tipo.

Proschan (1963) apontou que a heterogeneidade não observada pode explicar taxas de falhas crescentes, o que é frequentemente encontrado em análise de confiabilidade. Segundo Vaupel et al. (1979), os métodos padrões em análise de dados de sistemas reparáveis ignoram a heterogeneidade não observada. Assim, para contornar a questão da heterogeneidade, neste capítulo é proposto a modelo de reparo mínimo com fragilidade.

O modelo de fragilidade é caracterizado pela utilização de um efeito aleatório, isto é, uma variável aleatória não observável que representa as informações que não puderam ou não foram observadas, como fatores ambientais e climáticos, estresse de uso ou outras informações que por algum motivo não foram consideradas no planejamento, mas que de alguma forma são importantes para o estudo dos dados em sistemas reparáveis.

Uma das formas para incorporar esse efeito aleatório é introduzí-lo na função de intensidade com o objetivo de controlar a heterogeneidade não observável dos sistemas em estudo. A fragilidade pode ser inserida de forma multiplicativa ou aditiva a fim de avaliar o grau desta heterogeneidade entre os sistemas. Neste trabalho o termo de fragilidade 
atua de maneira multiplicativa na função de intensidade do modelo de reparo mínimo.

\subsection{Modelo de fragilidade multiplicativo}

$\mathrm{Na}$ literatura relacionada aos modelos de fragilidade diversos autores estudaram o uso dos modelos de fragilidade multiplicativos, sendo que estes representam uma extensão do modelo de Cox (Cox 1972). Do ponto de vista clássico, Andersen et al. (2012) e Hougaard (1995) apresentaram um revisão destes modelos, enquanto que Sinha e Dey (1997) elaboraram um revisão sob o enfoque bayesiano. Em dados univariados, o termo de fragilidade foi inserido por Vaupel et al. (1979) e Clayton (1978) e Oakes (1982) trabalham com os modelos para casos multivariados.

O modelo de fragilidade multiplicativo introduz um efeito aleatório, denominado fragilidade, na função de intensidade com o objetivo de descrever a possível associação existente entre as unidades, a fim de controlar a heterogeneidade não observável dos sistemas em estudo. Assim, o risco de um sistema depende de uma variável aleatória não observável, não negativa, a qual age de forma multiplicativa na função de intensidade.

Dessa forma, a intensidade de falha para o sistema $i$, para $i=1, \ldots, k$, é dada por

$$
\rho_{i}\left(t \mid z_{i}\right)=z_{i} \lambda_{0}\left(t_{i, j}\right)
$$

sendo que $\lambda_{0}\left(t_{i, j}\right)\left(j=1, \ldots, n_{i}\right)$ é a função de intensidade comum aos sistemas e $z_{i}$ é a variável de fragilidade independente e identicamente distribuida com função densidade $\mathrm{P}\left(z_{i}\right)$.

A função de confiabilidade condicional é estabelecida por

$$
R_{i}\left(t \mid z_{i}\right)=\left[R_{0}\left(t_{i, j}\right)\right]^{z_{i}}=\mathrm{e}^{\left[\Lambda_{0}\left(t_{i}^{*}\right) z_{i}\right]}
$$

em que $z_{i}$ representa a fragilidade do $i$-ésimo sistema, $\Lambda_{0}(t)$ é a função de intensidade acumulada comum à todas as unidades e $t_{i}^{*}$ é o truncamento por tempo, mas caso o estudo seja truncado por falha, basta fazer $t_{i}^{*}=t_{i, n_{i}^{*}}($ Seção 2.3).

Como $z_{i}$ representa um valor da variável aleatória não observável $Z$, quando $z_{i}>1$ significa que o risco individual é crescente e quando $z_{i}<1$ o risco é decrescente, já para $z_{i}=1$ o modelo de fragilidade (3.1) se reduz à função de intensidade comum às unidades.

Neste modelo, como a variável de fragilidade atua de forma multiplicativa, tem-se 
que quanto maior for o valor desta variável, maiores serão as chances de ocorrência de falha, ou seja, quanto maior for o valor de $z_{i}$, mais "frágeis" as unidades serão, daí o nome fragilidade. Portanto, para essas unidades mais frágeis é esperado que o evento de interesse ocorra mais frequentemente.

Em geral, assume-se que as fragilidades são independentes e identicamente distribuídas para cada unidade. Supondo que este termo de fragilidade segue uma distribuição de probabilidade, a escolha de tal distribuição é um ponto importante a ser abordado. Devido ao fato desse termo de fragilidade atuar de maneira multiplicativa na função de intensidade, as candidatas à distribuição de fragilidade são supostamente não negativas, usualmente contínuas e não dependentes do tempo, tais como distribuição gama, log-normal, gaussiana inversa e Weibull.

Devido ao fato da distribuição gama ser de fácil tratamento algébrico, ela tem sido muito utilizada para modelar a fragilidade. O primeiro modelo de fragilidade para dados de sobrevivência multivariado usando a distribuição gama foi considerado por Clayton (1978) e Oakes (1982).

Hougaard (1984) sugeriu para a variável fragilidade a distribuição gaussiana inversa como alternativa à distribuição gama, uma vez que essa distribuição torna a população mais homogênea com o passar do tempo, o que é compatível com a ideia de que o evento de interesse ocorre primeiramente para as unidades mais frágeis.

As distribuições gama e gaussiana inversa satisfazem as suposições de identificabilidade, na qual a fragilidade $Z$ é uma variável aleatória não negativa com função de distribuição $P(Z)$ e $E(Z)=1$ (Elbers e Ridder 1982). Do ponto de vista computacional, a distribuição gama ajusta-se muito bem à modelos de confiabilidade, porque é fácil derivar as fórmulas para alguns números de eventos.

Neste trabalho será considerada a distribuição gama, sendo assim, supondo que a variável de fragilidade tem distribuição Gama $(\alpha, \alpha)$, a função densidade de probabilidade para $Z$ é dada por

$$
f(z)=\frac{\alpha^{\alpha}}{\Gamma(\alpha)} z^{\alpha-1} \exp (-\alpha z)
$$

com valor esperado $E(Z)=1$, evitando assim os problemas de identificabilidade, e variância $V(Z)=1 / \alpha$, sendo que tal variância é parâmetro de dependência.

Observe que para $\alpha=0$, todas as variáveis de fragilidade são iguais a 1 e a distribuição 
gama fica degenerada no ponto 1 e, com isso, volta-se para o modelo de risco padrão para dados independentes.

A função de verossimilhança completa que contém os dados observados $\left(t_{i, j}\right)$ e não observados $\left(z_{i}\right)$ é dada por (veja Lawless (1987)):

$$
L\left(\boldsymbol{\mu}, z_{i}\right)=\prod_{i=1}^{k} \prod_{j=1}^{n_{i}} L\left(\boldsymbol{\mu} \mid z_{i}\right) P\left(z_{i}\right)
$$

em que a variável aleatória $z_{i}$ tem distribuição $P\left(z_{i}\right), \boldsymbol{\mu}$ é o vetor de parâmetros e $n_{i}$ é o número total de falhas observadas no $i$-ésimo sistema.

Considerando a função de verossimilhança do modelo de RM (2.18) e a inserção do termo de fragilidade multiplicativo da maneira como foi feita no modelo de fragilidade (3.1) e na função (3.2), pode-se reescrever a função (3.4) da seguinte forma:

$$
L\left(\boldsymbol{\mu}, z_{i}\right)=\prod_{i=1}^{k} \prod_{j=1}^{n_{i}}\left[z_{i} \lambda_{0}\left(t_{i, j}\right)\right] \exp \left\{-\Lambda_{0}\left(t_{i}^{*}\right) z_{i}\right\} P\left(z_{i}\right) .
$$

Integrando (3.5) em relação à $z_{i}$, sendo que $\mathrm{P}\left(z_{i}\right)$ é (3.3), a função de verossimilhança parcial marginal, tendo $n_{i}$ falhas observadas pelos tempos $t_{i, j}\left(j=1, \ldots, n_{i}\right)$ para o sistema $i$ é dada por:

$$
L_{i}=\int_{0}^{\infty}\left[\prod_{j=1}^{n_{i}} z_{i} \lambda_{0}\left(t_{j}\right)\right] \exp \left[\Lambda_{0}\left(t_{i}^{*}\right) z_{i}\right] \frac{\alpha^{\alpha}}{\Gamma(\alpha)} z_{i}^{\alpha-1} \exp (-\alpha z) d z_{i}
$$

A função (3.6) pode ser reescrita como

$$
\begin{aligned}
L_{i} & =\prod_{j=1}^{n_{i}}\left[\lambda_{0}\left(t_{i, j}\right)\right] \int_{0}^{\infty} z_{i}^{n_{i}} \frac{\alpha^{\alpha}}{\Gamma(\alpha)} \exp \left[\Lambda_{0}\left(t_{i}^{*}\right) z_{i}\right] z_{i}^{\alpha-1} \exp \left[-\alpha z_{i}\right] d z_{i} \\
& =\prod_{j=1}^{n_{i}}\left[\lambda_{0}\left(t_{i, j}\right)\right] \frac{\alpha^{\alpha}}{\Gamma(\alpha)} \int_{0}^{\infty} z_{i}^{n_{i}} z_{i}^{\alpha-1} \exp \left[\Lambda_{0}\left(t_{i}^{*}\right) z_{i}-\alpha z_{i}\right] d z_{i} \\
& =\prod_{j=1}^{n_{i}}\left[\lambda_{0}\left(t_{i, j}\right)\right] \frac{\alpha^{\alpha}}{\Gamma(\alpha)} \int_{0}^{\infty} \underbrace{-z_{i}^{n_{i}+\alpha-1} \exp \left[z_{i}\left(\Lambda_{0}\left(t_{i}^{*}\right)+\alpha\right)\right] d z_{i}}_{I}
\end{aligned}
$$

A parte $(I)$ da função (3.7) é muito similar a densidade de uma distribuição gama, 
exceto por uma constante. Então, pode-se reescrever esta função da seguinte maneira:

$$
\begin{aligned}
L_{i}= & \prod_{j=1}^{n_{i}}\left[\lambda_{0}\left(t_{i, j}\right)\right] \frac{\alpha^{\alpha}}{\Gamma(\alpha)} \frac{\Gamma\left(n_{i}+\alpha\right)}{\left(\Lambda_{0}\left(t_{i}^{*}\right)+\alpha\right)^{\left(n_{i}+\alpha\right)}} \\
& \int_{0}^{\infty} \underbrace{\frac{\left(\Lambda_{0}\left(t_{i}^{*}\right)+\alpha\right)^{\left(n_{i}+\alpha\right)}}{\Gamma\left(n_{i}+\alpha\right)} z_{i}^{n_{i}+\alpha-1} \exp \left[-z_{i}\left(\Lambda_{0}\left(t_{i}^{*}\right)+\alpha\right)\right.}_{I I}] d z_{i}
\end{aligned}
$$

Agora, note que a expressão (II) é a densidade de uma $\operatorname{Gama}\left(n_{i}+\alpha, \Lambda_{0}\left(t_{i}^{*}\right)+\alpha\right)$ e, então, sua integral é igual a um. Assim, a função de verossimilhança parcial marginal para o $i$-ésimo sistema é, portanto:

$$
\begin{aligned}
L_{i} & =\prod_{j=1}^{n_{i}}\left[\lambda_{0}\left(t_{i, j}\right)\right] \frac{\alpha^{\alpha}}{\Gamma(\alpha)} \frac{\Gamma\left(n_{i}+\alpha\right)}{\left(\Lambda_{0}\left(t_{i}^{*}\right)+\alpha\right)^{\left(n_{i}+\alpha\right)}} \\
& =\prod_{j=1}^{n_{i}}\left[\lambda_{0}\left(t_{i, j}\right)\right] \frac{\alpha^{\alpha} \Gamma\left(n_{i}+\alpha\right)}{\Gamma(\alpha)} \frac{1}{\left(\Lambda_{0}\left(t_{i}^{*}\right)+\alpha\right)^{\left(n_{i}+\alpha\right)}} \\
& =\prod_{j=1}^{n_{i}}\left[\lambda_{0}\left(t_{i, j}\right)\right] \frac{\alpha^{\alpha} \Gamma\left(n_{i}+\alpha\right)}{\Gamma(\alpha)}\left(\Lambda_{0}\left(t_{i}^{*}\right)+\alpha\right)^{-\left(n_{i}+\alpha\right)} \\
& =\prod_{j=1}^{n_{i}}\left[\lambda_{0}\left(t_{i, j}\right)\right] \frac{\alpha^{\alpha} \Gamma\left(n_{i}+\alpha\right)}{\Gamma(\alpha)}\left[\alpha\left(\frac{\Lambda_{0}\left(t_{i}^{*}\right)}{\alpha}+1\right)\right]^{-\left(n_{i}+\alpha\right)} \\
& =\prod_{j=1}^{n_{i}}\left[\lambda_{0}\left(t_{i, j}\right)\right] \frac{\alpha^{\alpha} \Gamma\left(n_{i}+\alpha\right)}{\Gamma(\alpha)} \alpha^{-\left(n_{i}+\alpha\right)}\left[\left(\frac{\Lambda_{0}\left(t_{i}^{*}\right)}{\alpha}+1\right)\right]^{-\left(n_{i}+\alpha\right)}
\end{aligned}
$$

Logo,

$$
L\left(\boldsymbol{\mu} \mid t_{i, j}\right)=\frac{\alpha^{-n_{i}} \Gamma\left(n_{i}+\alpha\right)}{\Gamma(\alpha)} \prod_{i=1}^{k} \prod_{j=1}^{n_{i}}\left[\lambda_{0}\left(t_{i, j}\right)\right]\left[\left(\frac{\Lambda_{0}\left(t_{i}^{*}\right)}{\alpha}+1\right)\right]^{-\left(n_{i}+\alpha\right)}
$$

Uma alternativa para determinar a função de verossimilhança marginal é obter a função de confiabilidade não condicional à fragilidade que, considerando a função (3.2), é preciso integrar o termo de fragilidade da seguinte maneira:

$$
R(t)=\int_{0}^{\infty} R_{i}\left(t \mid z_{i}\right) f(z) d z=\int_{0}^{\infty}\left[R_{0}(t)\right]^{z_{i}} f(z) d z=\int_{0}^{\infty}\left[\mathrm{e}^{-\Lambda_{o}\left(t^{*}\right)}\right]^{z_{i}} f(z) d z
$$

em que $f(z)$ é a função densidade de probabilidade da variável de fragilidade e $\Lambda_{0}(t)$ é a função de intensidade acumulada de $\lambda_{0}(t)$.

Para solucionar esta integral, a transformada de Laplace é a ferramenta de auxílio. 
Definição $A$ transformada de Laplace de um função $f:[0, \infty] \rightarrow R$ é definida por

$$
L_{f}(s)=E\left(\mathrm{e}^{-s t}\right)=\int_{0}^{\infty} \mathrm{e}^{-s t} f(t) d t
$$

para todo $s \geq 0$ em que a integral converge.

Considerando que $\mathrm{f}(\mathrm{t})$ tem distribuição Gama com a f.d.p (3.3), a transformada de Laplace da distribuição $\operatorname{Gama}(\alpha, \alpha)$ é dada por

$$
L_{g}[s]=\left(1+\frac{s}{\alpha}\right)^{-\alpha}
$$

Vê-se que as expressões (3.10) e (3.11) possuem a mesma forma. Então, na função (3.12) basta fazer $s=\Lambda_{0}(t)$ e dessa maneira, a função de confiabilidade condicional à fragilidade é determinada por

$$
R(t)=\left(1+\frac{\Lambda_{0}(t)}{\alpha}\right)^{-\alpha}
$$

E assim tem-se que a função de intensidade não condicional à fragilidade é dada por

$$
\rho(t)=\lambda_{0}(t)\left(1+\frac{\Lambda_{0}(t)}{\alpha}\right)^{-1}
$$

Portanto, sendo $t_{i, j}$ o $j$-ésimo tempo de falha do $i$-ésimo sistema, a partir das funções (3.13) e (3.14) obtidas pela transformada de Laplace, a função de verossimilhança parcial considerando o modelo de RM (2.18) é determinada por

$$
L\left(\boldsymbol{\mu} \mid t_{i, j}\right)=\prod_{i=1}^{k} \prod_{j=1}^{n_{i}}\left[\lambda_{0}\left(t_{i, j}\right)\right]\left[\left(\frac{\Lambda_{0}\left(t_{i}^{*}\right)}{\alpha}+1\right)\right]^{-\left(n_{i}+\alpha\right)}
$$

Observe que as função (3.9) e (3.15) são iguais a menos de uma constante, consequentemente, pode-se utilizar quaisquer uma das funções para estimar o parâmetro $\alpha$ a fim de quantificar a heterogeneidade não observável entre sistemas.

Considere a função de intensidade do modelo de fragilidade (3.1), cuja função de base $\lambda_{0}(t)$ seja a função (2.5). Desta forma é possível definir o modelo de interesse, o modelo reparo mínimo com fragilidade, cuja a função de intensidade do RM condicionada 
a variável de fragilidade é dada por

$$
\rho_{i}\left(t \mid z_{i}\right)=z_{i} \lambda_{0}(t)=z_{i} \beta \eta^{-\beta} t^{\beta-1}
$$

A função de confiabilidade do RM condicionada à variável $Z=z$ é estabelecida por

$$
R_{i}\left(t \mid z_{i}\right)=\left[R_{0}(t)\right]^{z_{i}}=\left[\exp \left(-\eta^{-\beta}\left(t^{*}\right)^{\beta}\right)\right]^{z_{i}}
$$

em que $z_{i}$ representa a fragilidade do $i$-ésimo sistema.

Com base na função (3.5), a função de verossimilhança para o modelo de reparo mínimo com fragilidade é, então, dada por

$$
L\left(\boldsymbol{\mu} \mid z_{i}\right)=\beta \eta^{-\beta} t_{i, j}^{\beta-1}\left[\exp \left(-\eta^{-\beta}\left(t_{i}^{*}\right)^{\beta}\right)\right]^{z_{i}} P\left(z_{i}\right)
$$

Como $P\left(z_{i}\right)$ é a função (3.3), a partir da função (3.15) tem-se que a função de verossimilhança para o modelo de reparo mínimo não condicionado a fragilidade é dado por:

$$
L(\boldsymbol{\mu})=\prod_{i=1}^{k} \prod_{j=1}^{n_{i}}\left[\beta \eta^{-\beta} t_{i, j}^{\beta-1}\right]\left(\frac{-\eta^{-\beta}\left(t_{i}^{*}\right)^{\beta}}{\alpha}+1\right)^{-\left(n_{i}+\alpha\right)}
$$

sendo que $t_{i}^{*}$ é o trucamento por tempo, mas se o estudo por truncado por falha, faça $t_{i}^{*}=t_{i, n_{i}^{*}}$, conforme descrito na Seção $(2.3)$.

O logaritmo da função de verossimilhança (3.19) é dado por

$$
\begin{aligned}
\ell_{R M}\left(\boldsymbol{\mu} \mid t_{i, j}\right)= & K N \log \left(\frac{\beta}{\eta}\right)+(\beta-1) \sum_{i=1}^{k} \sum_{j=1}^{n_{i}} \log \left(\frac{t_{i, j}}{\eta}\right)-\sum_{i=1}^{k} \sum_{j=1}^{n_{i}} \log \left(1+\frac{\left(\frac{t_{i}^{*}}{\eta}\right)^{\beta}}{\alpha}\right) \\
& -\alpha \sum_{i=1}^{k} \log \left(1+\frac{\left(\frac{t_{i}^{*}}{\eta}\right)^{\beta}}{\alpha}\right)
\end{aligned}
$$

em que $K$ é o número total de sistemas em estudo e $N=\sum_{i=1}^{k} n_{i}$, representando o número total de falhas ocorridas. Como não é possível obter uma solução analítica, métodos numéricos são considerados para a estimação dos parâmetros. 


\subsection{Estudo de Simulação}

No processo de simulação realizado, primeiramente, os parâmetros foram fixados em $\beta=50, \eta=17$ e $\alpha=7$, considerando um tipo de estudo truncado por falha, que neste caso foi considerado que os $k$ sistemas teriam 5 falhas.

Utilizou-se três tamanhos amostrais: $k=30, k=50$ e $k=100$ e foram geradas $d=1.000$ réplicas. A fim de otimizar o processo de simulação, os valores iniciais utilizados foram os mesmos valores pré-fixados para os parâmetros. Para atender os objetivos do estudo de simulação, utilizou-se o seguinte algoritmo:

1. Fixar os valores dos parâmetros;

2. Gerar $z_{i} \sim \operatorname{Gama}(\alpha, \alpha)$;

3. Gerar $u_{i} \sim U(0,1)$;

4. Gerar $t_{i, j}$, tal que $t_{i, j}=\left(\frac{-\log \left(1-u_{i}\right)}{z_{i}}\right)^{\frac{1}{\beta}} \eta$;

5. Repetir os passos do item 2 ao item $5 k$ vezes;

6. Repetir os passos 2 a 6 até obter $d$ réplicas.

A cada simulação foram obtidos o Erro Quadrático Médio (EQM) de cada estimativa, o Erro Padrão (EP), a amplitude e o intervalo de confiança de 95\%, baseado na teoria assintótica conforme determinado na simulação feita para o modelo ARA no Capítulo 3.

Os resultados obtidos estão resumidos na Tabela (3.1) e na Figura (3.1), em que EMV é o estimador de máxima verossimilhança. Os resultados mostram que conforme o tamanho da amostra aumenta, a amplitude do intervalo de confiança, o erro quadrático médio e a média dos desvios diminuem, como é esperado em um estudo de simulação para estimadores de máxima verossimilhança. Além disso, vê-se que a média dos valores estimados para os três parâmetros, $\beta, \eta$ e $\alpha$ aproximam-se dos valores fixados, conforme o tamanho das amostras aumentam, de acordo com o desejado para EMV. 
Tabela 3.1: Resultados do estudo de simulação do modelo de RM com fragilidade

\begin{tabular}{ccccccc}
\hline Amostras & Parâmetros & EMV & EP & EQM & IC $(95 \%)$ & Amplitude \\
\hline $\mathrm{k}=30$ & $\hat{\beta}$ & 52,596 & 3,930 & 6,317 & {$[44,893 ; 60,297]$} & 15,404 \\
& $\hat{\eta}$ & 12,546 & 0,039 & 0,234 & {$[12,401 ; 12,552]$} & 0,151 \\
& $\hat{\alpha}$ & 11,884 & 2,045 & 23,666 & {$[7,876 ; 15,891]$} & 8,015 \\
\hline $\mathrm{k}=50$ & $\hat{\beta}$ & 50,627 & 2,925 & 0,382 & {$[44,616 ; 56,083]$} & 11,467 \\
& $\hat{\eta}$ & 12,509 & 0,032 & 0,235 & {$[12,447 ; 12,574]$} & 0,127 \\
& $\hat{\alpha}$ & 7,873 & 0,726 & 0,010 & {$[5,482 ; 8,327]$} & 2,845 \\
\hline $\mathrm{k}=100$ & $\hat{\beta}$ & 50,350 & 2,131 & 0,119 & {$[46,449 ; 54,804]$} & 8,355 \\
& $\hat{\eta}$ & 12,508 & 0,023 & 0,188 & {$[12,462 ; 12,552]$} & 0,090 \\
& $\hat{\alpha}$ & 6,904 & 0,616 & 0,738 & {$[6,664 ; 9,080]$} & 2,416 \\
\hline
\end{tabular}
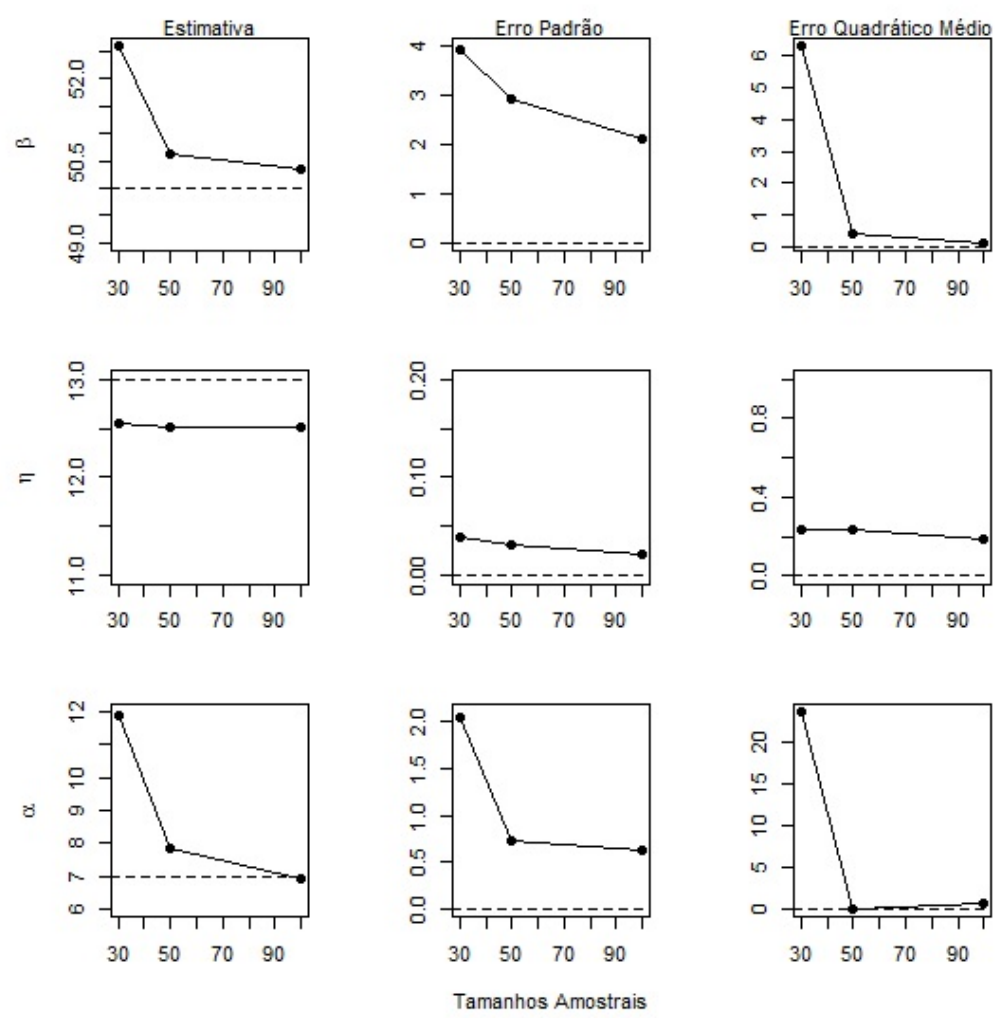

Figura 3.1: Gráfico dos Resultados da Simulação para o Modelo de RM com fragilidade 


\subsection{Aplicação aos dados dos caminhões basculantes}

A motivação deste trabalho é quantificar a heterogeneidade entre os caminhões basculantes da mineradora brasileira, descrito na Seção (2.5) cujo esboço dos dados foi apresentado na Tabela (2.2).

Foi utilizado o software $\mathrm{R}$ e a mesma rotina descrita na Seção (2.5), a fim de obter as estimativas dos parâmetros do modelo de RM com fragilidade. Os resultados seguem na Tabela (3.2), em que a estimativa para $\hat{\beta}=1,139$ e vê-se que o sistema está deteriorando (como já foi descrito) e o tempo médio esperado para a ocorrência de uma falha é de 6 dias $(\hat{\eta}=6,02)$ e como $\hat{\alpha}=42,82$, tem-se que a variabilidade é de $\frac{1}{42,82}=0,023$. Então, a variabilidade entre os caminhões é relativamente pequena, mas positiva, dando possíveis indícios da influência de fatores não observados.

Tabela 3.2: Estimativas para os parâmetros do modelo de RM com fragilidade via Máxima Verossimilhança e bayesiano

\begin{tabular}{cccc}
\hline Parâmetros & EMV & EP & IC $(0,95)$ \\
\hline$\hat{\beta}$ & 1,139 & 0,1012 & {$[0,940 ; 1,337]$} \\
$\hat{\eta}$ & 6,026 & 1,6428 & {$[2,806 ; 9,246]$} \\
$\hat{\alpha}$ & 42,892 & 6,1622 & {$[30,815 ; 54,970]$} \\
\hline
\end{tabular}

A qualidade do ajuste para o modelo de RM com fragilidade foi avaliada comparando as estimativas função de confiabilidade do modelo de reparo mínimo com fragilidade com Kaplan-Meier (KM) para eventos recorrentes, conforme procedimento descrito na Seção (2.5). O gráfico da Figura (3.2) apresenta as estimativas de Kaplan-Meier versus a função de confiabilidade estimada do modelo de reparo mínimo com fragilidade. Vê-se que o decaimento da confiabilidade estimada segue a curva de Kaplan-Meier, sugerindo que o ajuste parece adequado aos dados. 


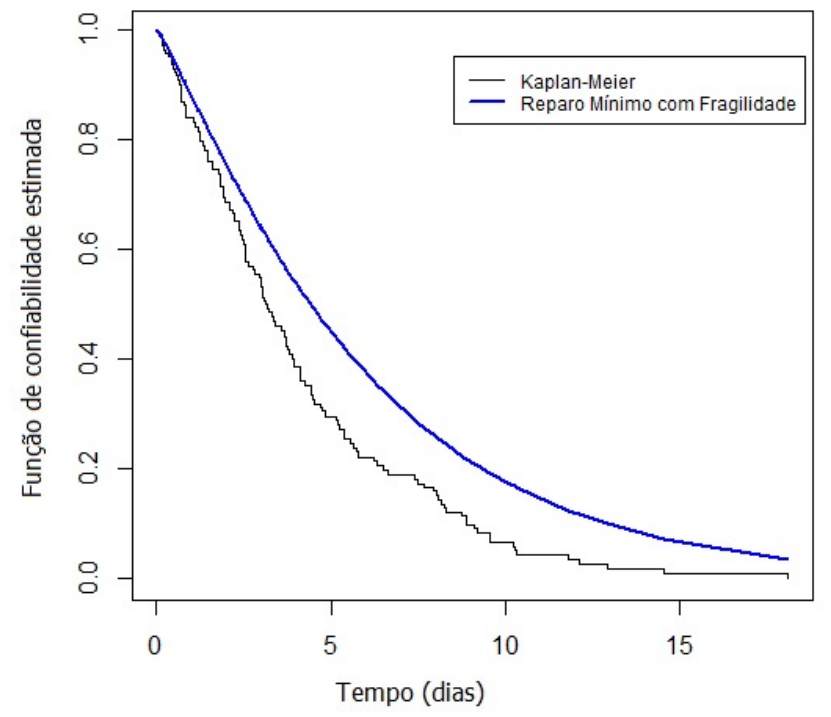

Figura 3.2: Gráfico de comparação de KM com função de confiabilidade estimada para o modelo de RM com fragilidade

Na Seção (2.5), foram obtidas as estimativas para o modelo de RM (que é o modelo sem fragilidade) e agora foram obtidas as estimativas para o modelo de RM com fragilidade. É de interesse verificar qual dentre esses dois modelos melhor se ajusta aos dados. Para tal finalidade, o critério de AIC (Akaike Information Criterion), BIC (Bayesian Information Criterion) e o logaritmo da verossimilhança foram utilizados. Tanto para o AIC quanto para o BIC, menores valores correspondem aos melhores modelos e pelo logaritmo da função de verossimilhança, o melhor modelo é aquele com a maior valor. Na Tabela 3.3 encontram-se os valores dos critérios AIC, BIC e a log-verossimilhança de cada modelo. Segundo estes critérios, o modelo escolhido é o modelo de RM (sem fragilidade).

Tabela 3.3: Critérios AIC, BIC e Log-verossimilhança dos modelos

\begin{tabular}{cccc}
\hline Modelo & AIC & BIC & Log-verossimilhança \\
\hline Sem Fragilidade & 618,3623 & 624,0819 & $-307,1811$ \\
Com Fragilidade & 685,8813 & 694,4607 & $-339,9406$ \\
\hline
\end{tabular}

O fato de o modelo de RM (sem o termo de fragilidade) ter sido o modelo selecionado, sugere que os caminhões são similares, mas isto será melhor abordado no capítulo seguinte, à partir das estimativas bayesianas da variável aleatória $Z$. 


\subsection{Alguns comentários}

Neste capítulo foi apresentado o modelo de interesse, que é o modelo de reparo mínimo com fragilidade. Os resultados do estudo de simulação estão de acordo com o esperado para estimadores de máxima verossimilhança e além disso, na aplicação ao conjunto de dados dos 5 caminhões basculantes, os resultados mostram que a variabilidade entre os caminhões é pequena, porém positiva, dando indícios de que é possível existirem fatores não observados que influenciam no tempo de falha.

O intuito do estudo também está em quantificar a fragilidade dos caminhões a fim de determinar qual, dentre eles, irá falhar antes dos demais. A estimação da variável $z_{i}$ é feita sob a abordagem bayesiana, que é apresentada na sequência, no Capítulo 4. 


\section{Capítulo 4}

\section{Método de estimação bayesiano}

A inferência bayesiana é uma alternativa para a estimação dos parâmetros. Neste capítulo, este método inferencial de estimação será considerado para obter as estimativas do modelo de reparo mínimo (visto no Seção 2.2.1) e do modelo de reparo mínimo com fragiliade (modelo proposto no Capítulo 3).

Sob o enfoque bayesiano, é possível expressar a incerteza a respeito dos parâmetros antes da observação dos dados, utilizando uma distribuição a priori para eles, enquanto que a distribuição à posteriori une a informação contida na verossimilhança com a distribuição à priori e, basicamente, as estimativas bayesianas são obtidas à partir da média desta distribuição à posteriori.

A distribuição à posteriori é determinada à partir do teorema de Bayes, que é dada por:

$$
\pi\left(\boldsymbol{\mu} \mid t_{i, j}\right) \propto L\left(\boldsymbol{\mu} / t_{i, j}\right) \pi(\boldsymbol{\mu})
$$

em que $L\left(\boldsymbol{\mu} / t_{i, j}\right)$ é a função de verossimilhança, $\boldsymbol{\mu}$ é o vetor de parâmetros e $t_{i, j}$ é o $j$-ésimo tempo de falha do $i$-ésimo sistema, para $i=1, \ldots, k$ e $j=1, \ldots, n_{i}$, sendo que $n_{i}$ é o número total de falhas do $i$-ésimo sistema.

Considerando a função de verossimilhança (2.19) do modelo de RM, tem-se que os parâmetros $\beta$ e $\eta$ assumem valores positivos e, portanto, é razoável considerar que $\beta \sim \operatorname{Gama}\left(a_{1}, b_{1}\right)$ e $\eta \sim \operatorname{Gama}\left(a_{2}, b_{2}\right)$ e admitir que $\beta$ e $\eta$ são independentes. Sendo assim, $\left(a_{1}, b_{1}\right)$ e $\left(a_{2}, b_{2}\right)$ são os hiperparâmetros do modelo. 
Dessa maneira, a distribuição à posteriori para o modelo de RM é dada por

$$
\pi_{R M}\left(\boldsymbol{\mu} \mid t_{i, j}\right) \propto \prod_{i=1}^{k} \prod_{j=1}^{n_{i}}\left[\beta \eta^{-\beta}\left(t_{i, j}\right)^{\beta-1}\right] \exp \left\{-\eta^{-\beta}\left(t_{i}^{*}\right)^{\beta}\right\} \beta^{a_{1}-1} e^{-\beta b_{1}} \eta^{a_{2}-1} e^{-\eta b_{2}}
$$

As distribuições condicionais completas para cada parâmetro do vetor $\boldsymbol{\mu}=(\beta, \eta)$ são dadas, respectivamente, por

$$
\pi_{R M}\left(\beta \mid t_{i, j}, \eta\right) \propto \prod_{i=1}^{k} \prod_{j=1}^{n_{i}}\left[\beta \eta^{-\beta}\left(t_{i, j}\right)^{\beta-1}\right] \exp \left\{-\eta^{-\beta}\left(t_{i}^{*}\right)^{\beta}\right\} \beta^{a_{1}-1} e^{-\beta b_{1}}
$$

$\mathrm{e}$

$$
\pi_{R M}\left(\eta \mid t_{i, j}, \beta\right) \propto \prod_{i=1}^{k} \prod_{j=1}^{n_{i}}\left[\beta \eta^{-\beta}\left(t_{i, j}\right)^{\beta-1}\right] \exp \left\{-\eta^{-\beta}\left(t_{i}^{*}\right)^{\beta}\right\} \eta^{a_{2}-1} e^{-\eta b_{2}}
$$

Vê-se que as condicionais completas parecem não seguir uma distribuição conhecida. Assim, a estimação deve ser feita utilizando um algoritmo de simulação, que neste trabalho foi considerado um algoritmo da classe MCMC (Markov Chain Monte Carlo).

Para o modelo de RM com fragilidade, tendo como base a função de verossimilhança (3.19) e tendo em vista que os parâmetros $\beta, \eta$ e $\alpha$ assumem valores positivos, é razoável tomar como distribuição à priori $\beta \sim \operatorname{Gama}\left(a_{1}, b_{1}\right), \eta \sim \operatorname{Gama}\left(a_{2}, b_{2}\right)$ e para $\alpha \sim \operatorname{Gama}\left(a_{3}, b_{3}\right)$. Assim, $\left(a_{1}, b_{1}\right),\left(a_{2}, b_{2}\right)$ e $\left(a_{3}, b_{3}\right)$ são os hiperparâmetros do modelo.

Logo, considerando que os três parâmetros são independentes, a distribuição à posteriori para o modelo de RM com fragilidade é determinada por:

$$
\begin{aligned}
\pi_{R M}\left(\boldsymbol{\mu} \mid t_{i, j}\right) \propto & \prod_{i=1}^{k} \prod_{j=1}^{n_{i}}\left\{\frac{\beta}{\eta}\left(\frac{t_{i, j}}{\eta}\right)^{\beta-1}\left(1+\frac{\left(\frac{t_{i}^{*}}{\eta}\right)^{\beta}}{\alpha}\right)^{-1}\right\}\left(1+\frac{\left[\left(\frac{t_{i}^{*}}{\eta}\right)^{\beta}\right]}{\alpha}\right)^{-\alpha} \\
& \beta^{a_{1}-1} e^{-\beta b_{1}} \eta^{a_{2}-1} e^{-\eta b_{2}} \alpha^{a_{3}-1} e^{-\alpha b_{3}} .
\end{aligned}
$$

As distribuições condicionais completas à posteriori para cada parâmetro do vetor $\boldsymbol{\mu}=(\beta, \eta, \alpha)$ são dadas por

$\pi_{R M}\left(\beta \mid t_{i, j}, \eta, \alpha\right) \propto \prod_{i=1}^{k} \prod_{j=1}^{n_{i}}\left\{\frac{\beta}{\eta}\left(\frac{t_{i, j}}{\eta}\right)^{\beta-1}\left(1+\frac{\left(\frac{t_{i}^{*}}{\eta}\right)^{\beta}}{\alpha}\right)^{-1}\right\}\left(1+\frac{\left[\left(\frac{t_{i}^{*}}{\eta}\right)^{\beta}\right]}{\alpha}\right)^{-\alpha} \beta^{a_{1}-1} e^{-\beta b_{1}}$ 
e

$$
\begin{aligned}
& \pi_{R M}\left(\eta \mid t_{i, j}, \beta, \alpha\right) \propto \prod_{i=1}^{k} \prod_{j=1}^{n_{i}}\left\{\frac{\beta}{\eta}\left(\frac{t_{i, j}}{\eta}\right)^{\beta-1}\left(1+\frac{\left(\frac{t_{i}^{*}}{\eta}\right)^{\beta}}{\alpha}\right)\right\}\left(1+\frac{\left[\left(\frac{t_{i}^{*}}{\eta}\right)^{\beta}\right]}{\alpha}\right)^{-\alpha} \eta^{a_{2}-1} e^{-\eta b_{2}} . \\
& \pi_{R M}\left(\alpha \mid t_{i, j}, \beta, \eta\right) \propto \prod_{i=1}^{k} \prod_{j=1}^{n_{i}}\left\{\frac{\beta}{\eta}\left(\frac{t_{i, j}}{\eta}\right)^{\beta-1}\left(1+\frac{\left(\frac{t_{i}^{*}}{\eta}\right)^{\beta}}{\alpha}\right)^{-1}\right\}\left(1+\frac{\left[\left(\frac{t_{i}^{*}}{\eta}\right)^{\beta}\right]}{\alpha}\right)^{-\alpha} \alpha^{a_{3}-1} e^{-\alpha b_{3}} .
\end{aligned}
$$

Nota-se, novamente, que as condicionais completas parecem não seguir uma distribuição conhecida. Assim, novamente, a estimação deve ser feita utilizando um algoritmo de simulação, que neste trabalho foi considerado um algoritmo baseado no método de simulação MCMC (Markov Chain Monte Carlo).

\subsection{Aplicação em dados reais}

Nesta secão é feita uma aplicação no conjunto de dados reais descrito na Seção(2.5) a fim de estimar os parâmetros dos modelos de reparo mínimo e do reparo mínimo com fragilidade sob o enfoque bayesiano.

Considerando a metodologia bayesiana descrita anteriormente e o conjunto de dados cujo esboço é dado na tabela (2.2), as estimativas dos parâmetros dos modelos em questão foram obtidas utilizando o software $\mathrm{R}$, à partir do pacote $M C M C p a c k$ pela rotina MCMCmetrop1R. Para mais detalhes sobre o algoritmo veja Gelman et al. (2003) e Robert e Casella (2013).

A distribuição gama foi considerada como distribuição à priori para os parâmetros do modelo de RM, isto é, $\beta \sim \operatorname{Gama}(0.01,0.01)$ e para $\eta \sim \operatorname{Gama}(1,0.1)$. Na simulação, foi utilizado um Burn-in de 100 valores e para eliminar possíveis autocorrelações foram utilizados, dos 100.000 simulados, os valores alternados de 10 em 10, totalizando uma amostra final de 10.000. A verificação da convergência gráfica segue na Figura (4.1), em que nota-se que para ambos os parâmetros houve convergência, pois, pelo gráfico dos traços, vê-se que estes estão em torno do valor estimado e pelo gráfico da densidade, 
nota-se que os valores estão mais concentrados em torno do valor estimado.
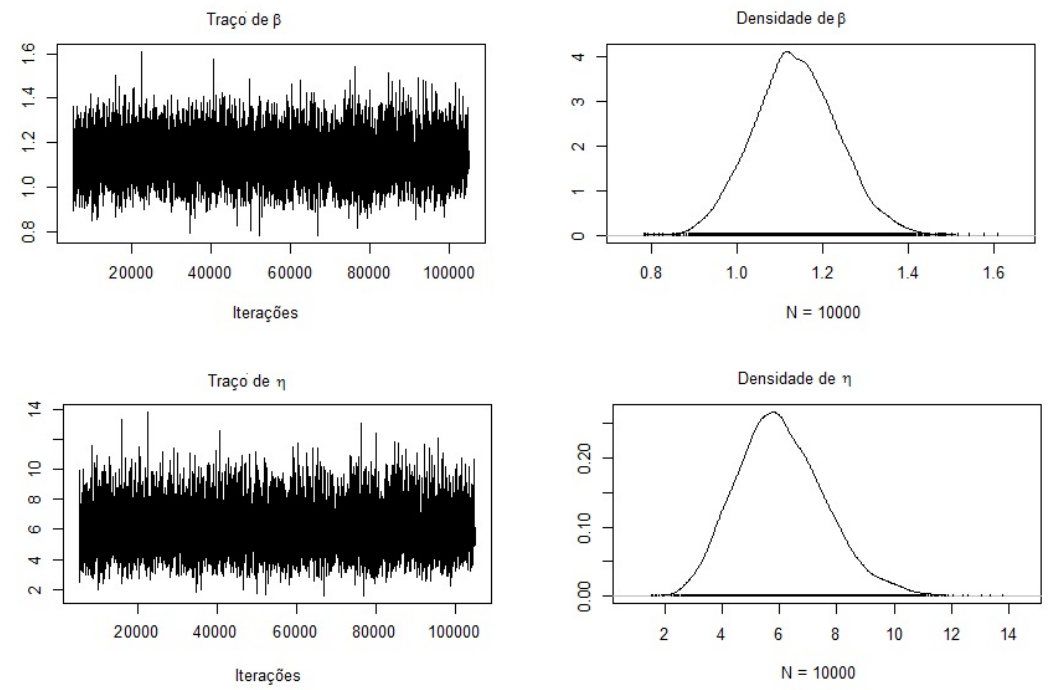

Figura 4.1: Convergência dos parâmetros $\beta$ e $\eta$ do modelo de RM

Também foi considerada a distribuição gama como distribuição à priori para os parâmetros do modelo de RM com fragilidade da seguinte maneira: $\beta \sim \operatorname{Gama}(0.01,0.01)$, $\eta \sim \operatorname{Gama}(1,0.1)$ e $\alpha \sim \operatorname{Gama}(20,1)$.

No procedimento de simulação a convergência iniciava apenas após os 400 primeiros valores, assim, foram descartados os primeiros 500 valores e no total foram simulados 300.000 valores, mas destes, foram considerados aqueles alternados de 50 em 50 valores, para evitar possíveis autocorrelações.

A convergência dos três parâmetros foram confirmadas a partir da análise gráfica, analogamente às interpretações anteriores, conforme Figura (4.2). 
Traço de $\beta$

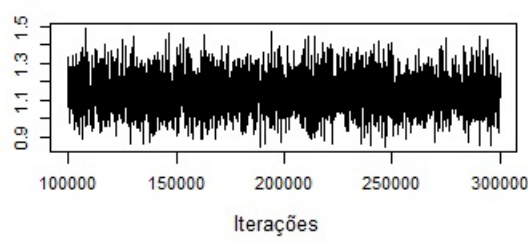

Traço de $\eta$

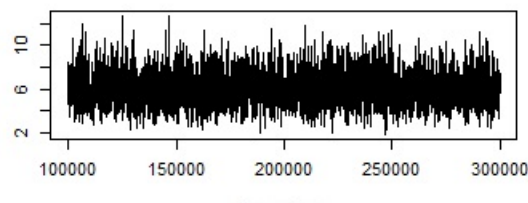

Iterações

Traço de o

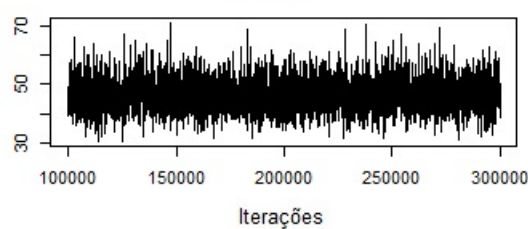

Densidade de $\beta$

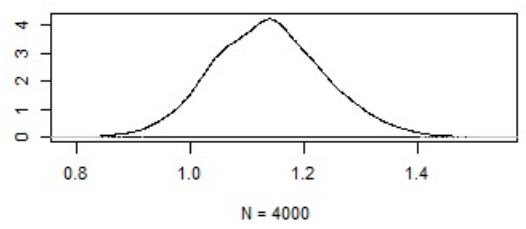

Densidade de $\eta$

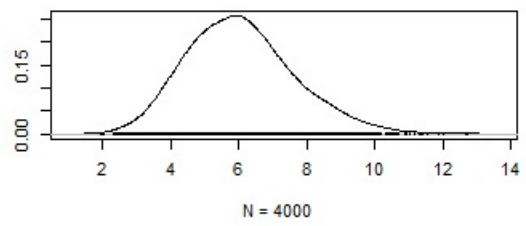

Densidade de $\alpha$

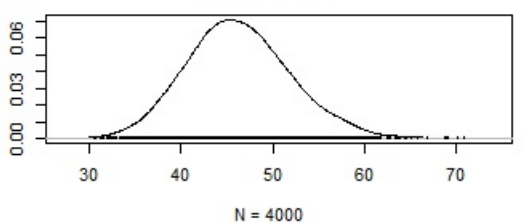

Figura 4.2: Convergência dos parâmetros $\beta, \eta$ e $\alpha$ do modelo de RM com fragilidade

Na Tabela (4.1) encontram-se os valores estimados segundo a média da distribuição à posteriori, em que observa-se que as estimativas bayesianas são similares às obtidas via máxima verossimilhança para ambos os modelos (Tabelas 2.3 e 3.2), denotando novamente que o sistema está deteriorando $(\hat{\beta}=1.13)$ e que tempo esperado para que ocorra uma falha é de 6 dias $(\hat{\eta}=6,0)$. Como $\hat{\alpha}=46,32$, tem-se que a variabilidade é de $\frac{1}{46,32}=0,0215$, portanto, a variabilidade entre os caminhões é relativamente pequena, mas positiva, dando indícios da possibilidade da influência de fatores não observados, conforme visto anteriormente.

Tabela 4.1: Estimativas obtidas pelo Método de Estimação Bayesiano

\begin{tabular}{cccccr}
\hline & Parâmetros & Média & EP & \multicolumn{2}{c}{$\operatorname{HPD}(95 \%)$} \\
\hline \multirow{2}{*}{$\mathrm{RM}$} & $\hat{\beta}$ & 1,138 & 0,09859 & {$[0,949 ;$} & $1,339]$ \\
& $\hat{\eta}$ & 6,062 & 1,5472 & {$[3,336 ;$} & $9,431]$ \\
\hline \multirow{3}{*}{ RM com fragilidade } & $\hat{\beta}$ & 1,137 & 0,09865 & {$[0,952 ;$} & $1,337]$ \\
& $\hat{\eta}$ & 6,057 & 1,58018 & {$[33,377 ;$} & $9,459]$ \\
& $\hat{\alpha}$ & 46,44 & 5,63757 & {$[36,507 ;$} & $58,327]$ \\
\hline
\end{tabular}




\subsubsection{Estimação à posteriori da a v.a. Z}

Como já foi mencionado, é de interesse estimar a fragilidade dos cinco caminhões basculantes a fim de sugerir o mais frágil dentre eles. Para isso, é necessário estimar a variável aleatória $z_{i}$ da função (3.16) e para tal, foram efetuados dois procedimentos.

O primeiro procedimento consiste no cálculo da mediana para os valores simulados da variável $z_{i}$ e no segundo, os valores de $z_{i}$ são obtido pela média à posteriori. Estes procedimentos são melhores apresentados na sequência.

A partir da estimativa do parâmetro $\hat{\alpha}=46,44$, tem-se que a variável aleatória $z_{i} \sim \operatorname{Gama}(46,46)$ e portanto, pode-se estimar a intensidade de falhas para cada um dos caminhões à partir da função (3.1) que é a função de intensidade do RM condicionada à fragilidade.

Primeiramente, foram gerados 1000 valores de $z_{i}$, para $i=(1,2,3,4,5)$. Então, foi calculada a mediana dos 1000 valores de $z_{i}$ para cada caminhão. Estes valores constam na Tabela (4.2).

Tabela 4.2: Mediana à posteriori simulados para a variável $Z$

\begin{tabular}{cccc}
\hline $\mathrm{Z}$ & Mediana & $\operatorname{HPD}(95 \%)$ \\
\hline$z_{1}$ & 0,988 & {$[0,883 ;$} & $1,195]$ \\
$z_{2}$ & 1,001 & {$[0,887 ;$} & $1,270]$ \\
$z_{3}$ & 1,001 & {$[0,864 ;$} & $1,257]$ \\
$z_{4}$ & 0,995 & {$[0,907 ;$} & $1,199]$ \\
$z_{5}$ & 0,980 & {$[0,885 ;$} & $1,212]$ \\
\hline
\end{tabular}

Dispondo dos valores medianos simulados de $z_{i}$ conforme Tabela (4.2) e dos valores estimados dos parâmetros obtidos pelo método bayesiano conforme Tabela (4.1) do modelo de RM com fragilidade, a função de intensidade para cada caminhão foi obtida considerando a função (3.16) e o gráfico referente à estes resultados segue na Figura (4.3). Nela, vê-se que as curvas do $2^{\circ}$ e do $3^{\circ}$ caminhão estão praticamente sobrepostas e são as curvas que indicam a maior intensidade de falhas, isto é, acredita-se que estes caminhões irão falhar antes dos demais. 


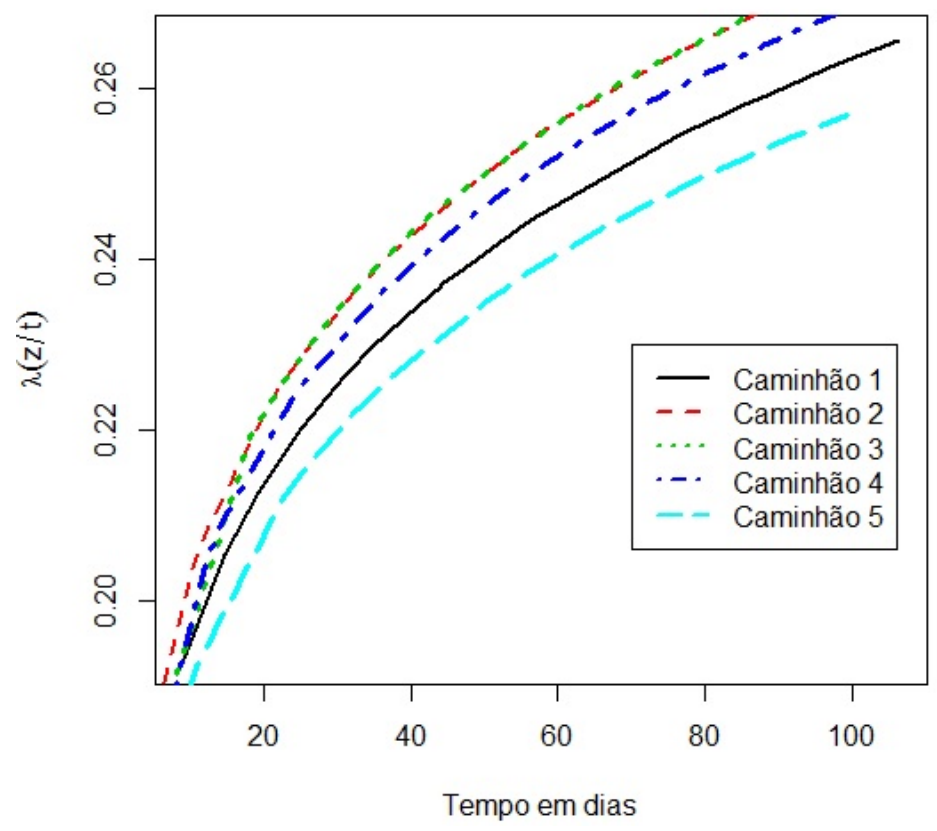

Figura 4.3: Função de intensidade condicionada à fragilidade para cada caminhão

O segundo procedimento para estimar a variável $Z$ é a partir de sua distribuição à posteriori. Considere a função de verossimilhança (3.4) para o modelo de RM com fragilidade e como distribuição à priori a distribuição $\operatorname{Gama}(\alpha, \alpha)$ para os valores de $z_{i}$. Então, a distribuição à posteriori condicional para $z_{i}$ dados $(\alpha, \beta, \eta)$ é dada por

$$
\pi\left(\boldsymbol{z}_{i} \mid t_{i, j}, \alpha, \beta, \eta\right) \propto z_{i}^{n_{i}+\alpha-1} \exp \left\{-z_{i}\left(\alpha+\eta^{-\beta} t_{i}^{* \beta}\right)\right\}
$$

Assim, vê-se que $\left(\boldsymbol{z}_{i} \mid t_{i, j}, \alpha, \beta, \eta\right) \sim \operatorname{Gama}\left(n_{i}+\alpha, \alpha+\eta^{-\beta} t_{i}^{* \beta}\right)$.

Neste caso, pode-se estimar $z_{i}$ via média à posteriori da distribuição $\left(\boldsymbol{z}_{i} \mid t_{i, j}, \alpha, \beta, \eta\right)$ que resulta em:

$$
\widehat{z_{i}}=\frac{n_{i}+\widehat{\alpha}}{\widehat{\alpha}+\widehat{\eta}^{-\widehat{\beta}} t_{i}^{* \widehat{\beta}}}
$$

em que as estimativas $\hat{\alpha}, \hat{\eta}, \hat{\beta}$ são os valores dispostos na Tabela 4.1 do modelo de RM com fragilidade.

Então, para o conjunto de dados dos 5 caminhões basculantes, os valores de $z_{i}$ calculados estão dispostos na Tabela (4.3) . Os resultados mostram que o $2^{\circ}$ caminhão é mais "frágil"' que os demais, portanto é esperado que ele venha a falhar antes dos outros caminhões. Pode-se dizer também que o $4^{\circ}$ caminhão irá presentar o evento de interesse 
antes dos caminhões 1,3 e 5, pois o valor obtido é maior que um, porém menor que o valor estimado para o $2^{\circ}$ caminhão.

Tabela 4.3: Média à posteriori para a variável $Z$

\begin{tabular}{cccc}
\hline Z & Média & \multicolumn{2}{c}{$\operatorname{HPD}(95 \%)$} \\
\hline$z_{1}$ & 0,965 & {$[0,751$,} & $1,205]$ \\
$z_{2}$ & 1,103 & {$[0,872$,} & $1,360]$ \\
$z_{3}$ & 0,976 & {$[0,759$,} & $1,218]$ \\
$z_{4}$ & 1,042 & {$[0,818$,} & $1,292]$ \\
$z_{5}$ & 0,991 & {$[0,771$,} & $1,237]$ \\
\hline
\end{tabular}

Contudo, observado os intervalos de credibilidade HPD tanto para a mediana (Tabela 4.2) quanto para a média (Tabela 4.3) dos valores de $z_{i}$, vê-se que o valor 1 está contido nos intervalos, indicando que os caminhões são similares, pois, apesar de existir alguma diferença entre eles, está não é significativa.

Portanto, apesar dos indícios dados pela estimativa de $\alpha$ de que poderiam haver fatores externos não observados que deveriam ter sido considerados e os indícios de que o $2^{\circ}$ caminhão é mais frágil que os demais, pelo intervalo HPD pode-se dizer que fatores externos não influenciam no tempo de falha dos 5 caminhões basculantes, o que vai ao encontro do estudo feito por Toledo (2014).

\subsection{Alguns comentários}

Neste capítulo foram estudados os modelos de reparo mínimo com e sem fragilidade sob o enfoque bayesiano, em que viu-se que os estimativas obtidas são similares as encontradas via máxima verossimilhança e, consequentemente, suas interpretações também. Além disso, foram estimados os valores da variável aleatória $Z$ a fim de quantificar a fragilidade individual dos caminhões e as análises feitas por meio do intervalo de credibilidade mostram que os caminhões são similares. 


\section{Capítulo 5}

\section{Considerações finais e propostas}

\section{futuras}

Neste trabalho, foi feita uma breve revisão dos processos de contagem, incluindo os processos de Poisson não homogêneo, processo de renovação e a forma paramétrica do processo de lei de potência. Foi feita uma introdução aos sistemas reparáveis, destacando as principais diferenças entre os sistemas reparáveis e não reparáveis. Os modelos mais conhecidos na literatura foram apresentados, sendo eles o modelo de reparo mínimo, de reparo perfeito e de reparo imperfeito e foram mostrados o conceito de idade virtual e as classes de modelos ARA e ARI.

Um estudo de simulação para o modelo ARA foi realizado, cujos resultados estão de acordo com o esperado para estimadores de máximoa verossimilhança e uma aplicação em um conjunto de dados reais foi realizada a fim de mostrar a aplicabilidade dos modelos de reparo mínimo, ARA e ARI.

Os principais conceitos de fragilidade foram apresentados e o modelo de interesse, modelo de reparo mínimo com fragilidade, foi apresentado. Além disso, um estudo de simulação foi realizado para o modelo proposto, sendo que os resultados obtidos estão de acordo com o esperado para estimadores de máxima verossimilhança e na aplicação aos dados dos 5 caminhões basculantes da mineradora brasileira, pode-se mensurar a heterogeneidade não observada.

Sob o enfoque bayesiano, as estimativas foram obtidas para o modelo de reparo mínimo e para o modelo de reparo mínimo com fragilidade, em que também foi possível verificar se os 5 caminhões são similares ou não.

Para trabalhos futuros propõe-se considerar outras formas paramétricas que não a 
do processo de lei de potência para os modelos de reparo mínimo, perfeito e imperfeito, e propõe-se considerar também a modelagem de fragilidade para os modelos de reparo perfeito e imperfeito e sugere-se a inclusão de covariáveis observáveis na modelagem proposta. 


\section{Referências Bibliográficas}

Andersen, P. K.; Borgan, O.; Gill, R. D.; Keiding, N. Statistical models based on counting processes. Springer Science \& Business Media, 2012.

Ascher, H.; Feingold, H. Repairable systems reliability: modeling, inference, misconceptions and their causes. M. Dekker New York, 1984.

Brown, M.; Proschan, F. Imperfect repair. Journal of Applied probability, pages 851-859, 1983.

Clayton, D. G. A model for association in bivariate life tables and its application in epidemiological studies of familial tendency in chronic disease incidence. Biometrika, v. $65(1)$, p. 141-151, 1978.

Colosimo, E. A.; Giolo, S. R. Análise de sobrevivência aplicada. In ABE-Projeto Fisher. Edgard Blücher, 2006.

Cox, D. R. Regression models and life tables (with discussion). Journal of the Royal Statistical Society, Series B, v. 34(2), 1972.

Crow, L. H. Reliability analysis for complex, repairable systems. in reliability and biometry, ads. f. proschan and r. j. serfling, philadelphia. Technical report, DTIC Document, 1974.

Doyen, L.; Gaudoin, O. Classes of imperfect repair models based on reduction of failure intensity or virtual age. Reliability Engineering 83 System Safety, v. 84(1), p. $45-56,2004$.

Elbers, C.; RIDDER, G. True and spurious duration dependence: The identifiability of the proportional hazard model. The Review of Economic Studies, v. 49(3), p. 403-409, 1982. 
Gelman, A.; Carlin, J. B.; Stern, H. S.; Rubin, D. B. Bayesian data analysis, (chapman \& hall/crc texts in statistical science). 2003.

HougaARD, P. Life table methods for heterogeneous populations: distributions describing the heterogeneity. Biometrika, v. 71(1), p. 75-83, 1984.

HougaArd, P. Frailty models for survival data. Lifetime data analysis, v. 1(3), p. 255-273, 1995.

KiJima, M. Some results for repairable systems with general repair. Journal of Applied probability, pages 89-102, 1989.

Kijima, M.; Morimura, H.; Suzuki, Y. Periodical replacement problem without assuming minimal repair. European Journal of Operational Research, v. 37(2), p. 194-203, 1988.

Lawless, J. F. Regression methods for poisson process data. Journal of the American Statistical Association, v. 82(399), p. 808-815, 1987.

Lindqvist, B. H. On the statistical modeling and analysis of repairable systems. Statistical science, pages 532-551, 2006.

Muralidharan, K. A review of repairable systems and point process models. In ProbStat Forum, volume 1, pages 26-49, 2008.

Nelder, J. A.; Mead, R. A simplex method for function minimization. The computer journal, v. 7(4), p. 308-313, 1965.

OAKES, D. A model for association in bivariate survival data. Journal of the Royal Statistical Society. Series B (Methodological), pages 414-422, 1982.

Oliveira, M. D. D.; Colosimo, E. A.; Gilardoni, G. L. Power law selection model for repairable systems. Communications in Statistics-Theory and Methods, v. 42(4), p. $570-578,2014$.

Proschan, F. Theoretical explanation of observed decreasing failure rate. Technometrics, v. 5(3), p. 375-383, 1963.

Rigdon, S. E.; BAsu, A. P. Statistical methods for the reliability of repairable systems. Wiley New York, 2000. 
Robert, C.; Casella, G. Monte Carlo statistical methods. Springer Science \& Business Media, 2013.

SinhA, D.; Dey, D. K. Semiparametric bayesian analysis of survival data. Journal of the American Statistical Association, v. 92(439), p. 1195-1212, 1997.

Toledo, M. L. G. D. Determination of the optimal periodic maintenance policy under imperfect repair assumption. Tese (Doutorado), Escola de Engenharia - Departamento de Engenharia da Prodũ̃ß̧ao, 2014.

Vaupel, J. W.; Manton, K. G.; Stallard, E. The impact of heterogeneity in individual frailty on the dynamics of mortality. Demography, v. 16(3), p. 439-454, 1979.

Wang, M.-C.; Chang, S.-H. Nonparametric estimation of a recurrent survival function. Journal of the American Statistical Association, v. 94(445), p. 146-153, 1999. 